\title{
Large-scale sea ice drift and deformation: Comparison between models and observations in the western Weddell Sea during 1992
}

\author{
Cathleen A. Geiger \\ University Space Research Association, NASA Goddard Space Flight Center, Greenbelt, Maryland
}

William D. Hibler III ${ }^{1}$

Institute of Geophysics, University of Texas at Austin

Stephen F. Ackley

Snow and Ice Branch, Cold Regions Research and Engineering Laboratory, Hanover, New Hampshire

\begin{abstract}
Statistical comparisons between numerical sea ice models and an observed large-scale strain array in the western Weddell Sea during 1992 are used to evaluate the performance of three of the more generally utilized sea ice rheology formulations. Results show that sea ice velocity is reproduced with relatively high accuracy $(90 \%$ coherence, $>80 \%$ normalized cross correlation) in models having high-quality atmospheric forcing fields (e.g., the European Centre for Medium-Range Weather Forecasts). On the other hand, temporal and spatial variability of the velocity field, as exemplified by progressive vector plots and ice deformation, respectively, are reproduced less accurately (coherence and normalized cross correlation $<50 \%$ ). In terms of model sensitivity, this means that deformation and temporal variability are more discriminating in terms of elucidating specifics about the constitutive relation and mechanical properties of sea ice on a large scale. For example, inclusion of both compressive and shear stresses is important in attaining a proper probability distribution of deformation relative to observations. Additional analysis shows that adjustments to specific model parameters improve the model results for either drift or select deformation components, but no best solution could be found, given the models examined here. Results suggest that inclusion of more physically based processes, such as subdaily tidal and inertial oscillations, reconsideration of the boundary layer formulation, and consideration of anisotropy, may be necessary to include in next-generation sea ice models, especially those that are intended for coupling with high-resolution (eddy resolving) ocean models.
\end{abstract}

\section{Introduction}

Reproduction of observed large-scale physical features and processes in sea ice is a critical test of model performance. This is particularly true of regional to global scale models where forecasting is involved. Prognosis of future conditions without physically based benchmarks is, at best, speculation. A fundamental question relevant to this issue is, what are the critical variables

\footnotetext{
${ }^{1}$ On leave from Thayer School of Engineering, Dartmouth College, Hanover, New Hampshire.
}

Copyright 1998 by the American Geophysical Union.

Paper number 98JC01258.

0148-0227/98/98JC-01258\$09.00 of sea ice that are both robust enough to be monitored easily on a large scale and yet sensitive enough to indicate a change in air-ice-sea circulation? In terms of the large-scale physics of the problem, if we consider the air-ice-sea system deterministically, sea ice responds uniquely to atmospheric and oceanic forces through four measurable variables: sea ice thickness, areal extent/compactness, drift, and deformation.

Basically, atmospheric and oceanic forcing serve as inputs to initiate sea ice response through a set of coupled equations that include a sea ice mass balance, momentum balance, and constitutive relation (see, for example, Hibler [1979] for mathematical formulation). The four sea ice parameters of thickness $h$, compactness $A$, velocity $\mathbf{v}$, and deformation components $\dot{\epsilon}_{2 j}$ can be regarded as responses to the imposed forcing (i.e., outputs 
resulting from the imposed forcing). While a number of feedback processes ensue between the air, ice, and ocean owing to the nonlinear response of all three, these four outputs provide large-scale, physically measurable field quantities resulting from air-ice-sea activity. Hence changes transmitted between air and ocean in the polar regions must pass through the sea ice/open water interface, with measurable changes reflected in the above four sea ice parameters.

In terms of their relative contributions, ice thickness describes the vertical extent of the ice while compactness deals with the horizontal area and extent. Velocity describes the average flow of ice, while deformation expresses the structural integrity of the field and spatial variability of the ice motion. All four are needed to accurately describe and reproduce the physical conditions of sea ice on a large scale.

Recent work by Geiger et al. [1997] has examined the thermodynamic response of ice edge extent and thickness distribution to various atmospheric and oceanic forcing terms. Complementing that study and building upon results from Hibler and Ackley [1983], we present here a numerical investigation of the large-scale mechanical responses of the ice. Specifically, we focus on observed versus modeled drift and strain-rate responses to determine how well large-scale sea ice models reproduce these variables. Dynamic-thermodynamic sea ice models using viscous-plastic (VP) and cavitating fluid (CAV) ice models will be examined, as these are widely used in both sea ice models and coupled air-ice and iceocean studies, particularly the cavitating fluid model which is used in conjunction with climate models. Together with the free drift solution, they also provide a logical hierarchy of sea ice rheologies from free drift (no resistance) through cavitating fluid (pressure resistance) to viscous plastic (pressure and shear resistance).

As seen in the work by Geiger et al. [1997] and Geiger [1996], ice edge location is not very sensitive in these models compared to ice thickness distribution and compactness. Additionally, ice thickness spatial distribution is not a good parameter to examine sensitivity to internal ice variables [Geiger, 1996], primarily for two reasons. First, while variations in internal ice variables can be used to address the issue of how certain models affect ice thickness and compactness, it is very difficult to decipher why this is the case and what are the underlying processes responsible. In other words, it is difficult to deduce what is happening physically to produce such a result. Second, there is currently a lack of regionally observed thickness distributions for comparison. This makes it extremely difficult to test which thickness distribution field is correct and, furthermore, which set of internal ice parameters is correct for that observed thickness distribution. The same argument holds more or less for compactness. The end result is that we need a better method for examining responses of the internal ice interaction in order to understand the physics of the observed sea ice mechanics.
Statistical evaluation of individual buoy tracks has provided a more realistic source for comparing results between different rheology model types [e.g., Hibler and $I p, 1995]$, but this is only part of the information necessary to test these models. The distinguishing characteristic in sea ice models is the constitutive relation. Therefore a rigorous examination of internal ice interaction must include direct examination of both drift (velocity) and deformation. Drift can be thought of as the mean motion of the ice, while deformation is its spatial variability. Obtaining the right mean in a model is a good first-order result, but variability is a more sensitive and defining factor in terms of isolating the correct physics. Deformation is also a more critical factor than drift in terms of the thermodynamic balance because the open water fraction created by deformation processes is the greatest contributor to heat and mass (salt expulsion) transfer in polar regions [Maykut, 1986]. Like the coupling between thickness, compactness, velocity, and deformation, the physical processes that make up deformation itself are also coupled. Hence, in order to understand how the open water fraction of ice is formed, one must also understand how processes such as shear and divergence work in conjunction with each other. For this reason, shear is considered as important as divergence for determining the underlying physics.

The purpose of this study is to better understand the physics of sea ice as described by the numerical models indicated above and to determine how these models compare with observations. A unique data set from Ice Station Weddell (ISW) in the austral fall of 1992 in the western Weddell Sea using six buoys in a large-scale array provides the opportunity to test models against observed drift and deformation. An overview of the field experiment is given in section 2, with particular emphasis on drift and deformation characteristics of the region. This is followed by a scaling analysis of the momentum balance to illustrate the significance of deformation as a means of understanding the variability of sea ice motion. Next, a description of the numerical schemes and details of the ice rheologies are presented. This is followed by results from comparison studies between models and observations using crossspectral analyses, cross correlation, $\chi^{2}$ analyses, and probability density distribution of drift and deformation components. These methods compare modeled and observed buoy motion in the time domain, the frequency domain, and with respect to distribution categories in principal axis space and scatterplots. Since the primary goal is to compare sea ice drift and deformation between models and observations, the impact on thickness and compactness is left for future research.

\section{Field Experiment}

During ISW 1992 [Gordon et al., 1993], a large-scale drifting Argos buoy array spanning $150 \mathrm{~km}$ was set up in the western Weddell Sea. Five Argos buoys at re- 


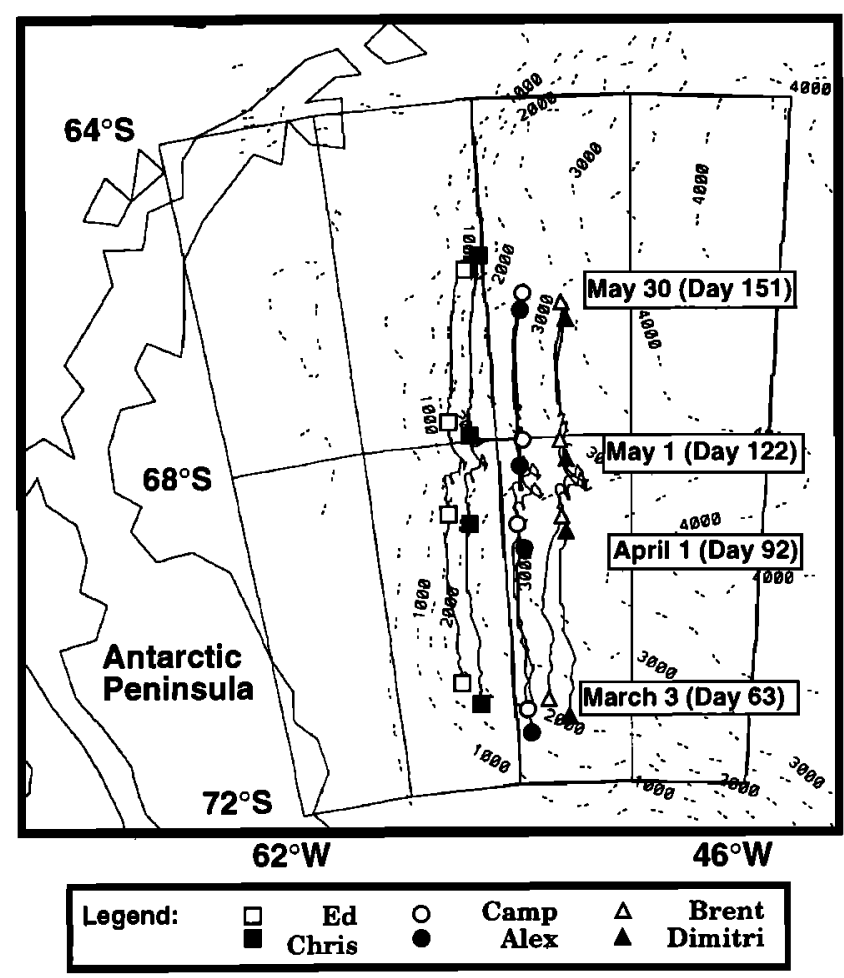

Figure 1. Western Weddell Sea region with Ice Station Weddell (ISW) buoy tracks and local bathymetry.

mote sites located 25 to $100 \mathrm{~km}$ from the central camp recorded geographical position about every 3 hours via satellite, while a Global Positioning System (GPS) unit at the camp recorded geographical positions about 20 times per day. Figure 1 shows the general track of the buoys, their relative configuration within the array, and the local bathymetry. Using a Butterworth low-pass filter and multiple linear regression, the array position data were processed [Geiger et al., 1998] to identify drift and strain-rate components.

Details of these results are presented by Geiger et al. [1998] and Geiger [1996]. As a synopsis, the power spectra results from ISW wind, sea ice, and ocean current measurements together with deformation analysis of the sea ice reveal the following. First (Figure 2, left center), sea ice velocity in the western Weddell region is a low-frequency $\left(<1\right.$ cycle $\left.\mathrm{d}^{-1}\right)$ dynamic process driven primarily by low-frequency forcing in the form of moderate, steady ocean currents and intermittent strong winds from high-energy storm activity. Second, higher frequencies, specifically diurnal and semidiurnal tidal/inertial oscillation frequencies, form the main contribution to sea ice deformation (ice total strain rate; Figure 2, right center) in this region. Shear deformation has large high- and low-frequency components, with elongation (normal deformation) oriented parallel to the shelf break being the main form of deformation at low frequencies. The observed higher-frequency processes correlate with 12 and 24 hour ( 2 and 1 cycle $\mathrm{d}^{-1}$ ) ocean oscillations (Figure 2, bottom), with a 12 hour peak contributing the most to the total shear activity and the 24 hour peak contributing more to the solid body rotation (vorticity) of ice on scales as large or larger than the ISW array $(150 \mathrm{~km})$. East to west rising ocean bottom topography of the continental slope is also believed to play a major role in the directional preference of both observed ice drift and deformation in this region. Ice drift and deformation seem particularly sensitive to the forcing caused by topographic change, as enhanced by ocean currents.

\section{Drift and Differential Drift Scaling}

The momentum balance of sea ice may be written

$$
m \frac{D \mathbf{v}}{D t}=-m f \mathbf{k} \times \mathbf{v}+\tau_{a}+\tau_{w}-m g \nabla H+\mathbf{F}_{\text {ice }}
$$

where $m$ is ice mass; $\mathbf{v}$ is ice velocity vector; $f$ is the Coriolis term; $\mathbf{k}$ is the unit vector in the vertical; $\tau_{a}$ and $\tau_{w}$ are the forces from air and water stresses, respectively; $\mathbf{F}_{\text {ice }}$ is the ice interaction force; $g$ is gravity; and $H$ is sea surface height. If we consider sea ice motion over a region where deformation is occurring, the spatial differential drift or deformation may be viewed as a perturbation on a more smoothly varying drift. As a consequence to lowest order, we may consider the nonlinear water drag and wind drag to be slowly varying over a deformation region on the scale of about 100 $\mathrm{km}$. With these approximations in mind and using a procedure similar to that of Hibler et al. [1974], we may take derivatives of the momentum equation, obtaining relationships for the various kinematical quantities. In particular, if we consider an approximation to (1) whereby the inertial terms on the left-hand side vanish and no ocean current is assumed, we can differentiate the $x$ and $y$ component equations by $\partial / \partial y, \partial / \partial x$, respectively, and sum to form (2) and $\partial / \partial x,-\partial / \partial y$ for (3). In this way, we form coupled equations for the local shear deformation (SD) and so-called normal deformation (ND) (or local elongation).

$$
\begin{aligned}
C_{w, d} \mathrm{SD}_{i} & +\left(C_{w, a}+m f\right) \mathrm{ND}_{i} \\
& =C_{a, d} \mathrm{SD}_{a}+C_{a, a} \mathrm{ND}_{a}+\frac{\partial F_{x}}{\partial x}+\frac{\partial F_{y}}{\partial y} \\
C_{w, d} \mathrm{ND}_{i} & -\left(C_{w, a}+m f\right) \mathrm{SD}_{\imath} \\
& =C_{a, d} \mathrm{ND}_{a}-C_{a, a} \mathrm{SD}_{a}+\frac{\partial F_{x}}{\partial x}-\frac{\partial F_{y}}{\partial y}
\end{aligned}
$$

where $C_{a, d}=C_{a}^{\star} \cos \theta$ is the air diagonal term, $C_{a, a}=$ $C_{a}^{\star} \sin \theta$ is the air asymmetric term, $C_{a}^{\star}$ is the air drag coefficient, and analogously, $w$ is for ocean, $\theta$ is turning angle, $F_{x}$ and $F_{y}$ are ice interaction terms in $x$ and $y$ directions, respectively and $\mathrm{SD}_{2}, \mathrm{ND}_{i}, \mathrm{SD}_{a}$, and $\mathrm{ND}_{a}$ are shear and normal deformation for ice and air, respectively; defined as 

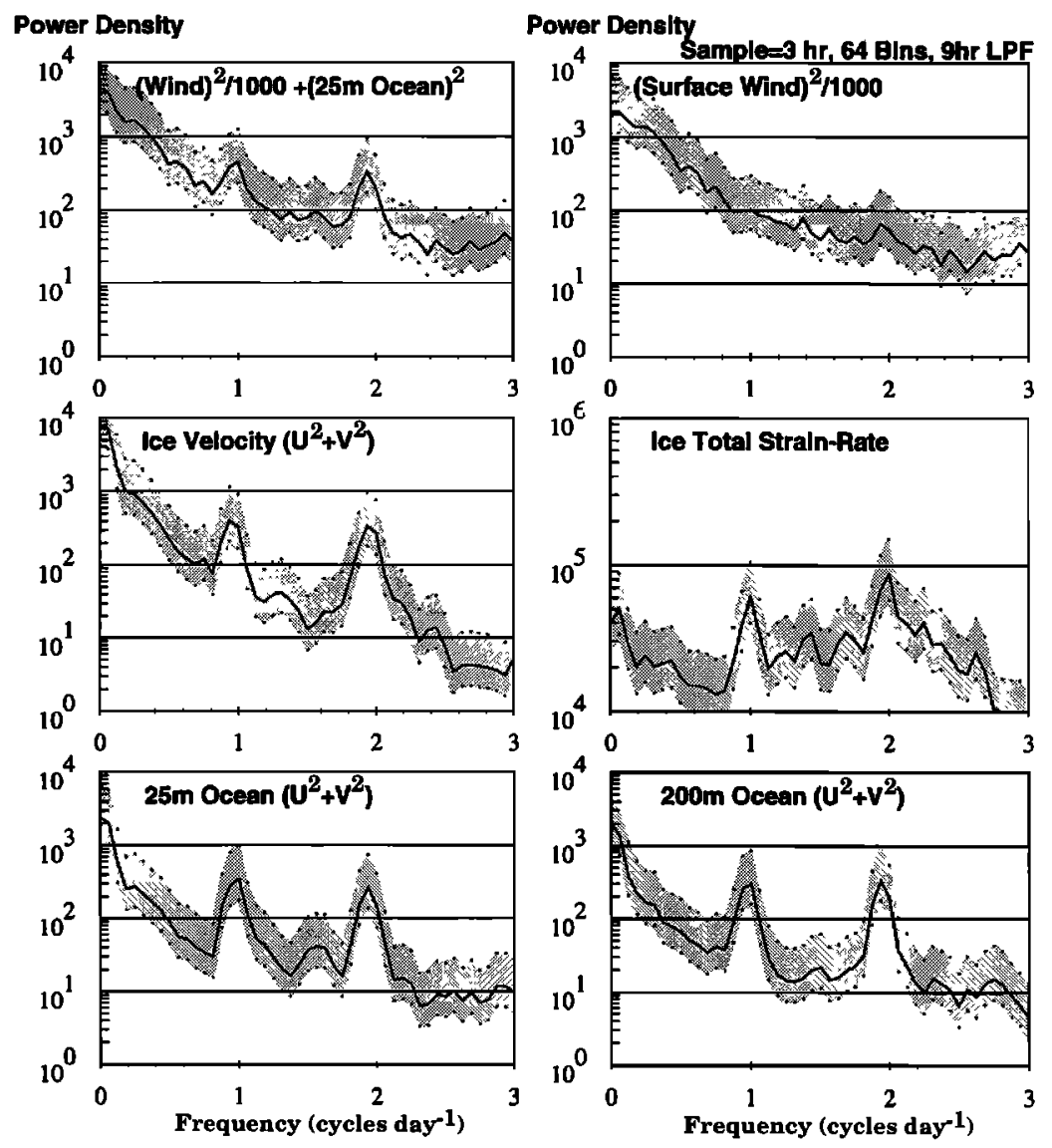

Figure 2. Power density spectra from the observed ISW large-scale strain array for surface wind, ice, 25 and $200 \mathrm{~m}$ water velocities, and total ice strain rate resolved to 64 frequency bins. Power density of wind, ice, and ocean is in square meters per second, and strain rate is in units of $\times 10^{-12} \mathrm{~s}^{-1}$. The wind power (velocity squared) is divided by 1000 to compare it with the kinetic energy in the ocean by compensating for the differences in density of the two media. The $90 \%$ confidence interval of $\chi^{2}$ results are shown in shaded regions.

$$
\begin{aligned}
\mathrm{SD} & =\frac{\partial v}{\partial x}+\frac{\partial u}{\partial y}=2 \dot{\epsilon}_{x y} \\
\mathrm{ND} & =\frac{\partial u}{\partial x}-\frac{\partial v}{\partial y}=\dot{\epsilon}_{x x}-\dot{\epsilon}_{y y}
\end{aligned}
$$

Similar coupled equations can be derived for divergence and vorticity.
Using results from ISW, we obtain the observed ice kinematic quantities found in (1)-(3). Using the full nonlinear water and air drag terms, we compute these terms for the free drift case with no ocean. Table 1 shows the variance and rms values of the velocity magnitude and the differential drift values for both of these cases. Table 1 demonstrates that while the magnitudes

\begin{tabular}{|c|c|c|c|c|c|c|c|c|c|c|}
\hline & \multirow{2}{*}{\multicolumn{2}{|c|}{ Observed }} & \multicolumn{4}{|c|}{$\mathrm{FD}$} & \multirow{2}{*}{\multicolumn{2}{|c|}{ CAV }} & \multirow{2}{*}{\multicolumn{2}{|c|}{ VP TRU }} \\
\hline & & & \multicolumn{2}{|c|}{ No Ocean Current } & \multicolumn{2}{|c|}{ Ocean Current } & & & & \\
\hline & variance & $\mathrm{rms}$ & variance & $\mathrm{rms}$ & variance & rms & variance & rms & variance & $\mathrm{rms}$ \\
\hline VM & 48.32 & 11.92 & 55.03 & 13.94 & 59.25 & 14.07 & 61.79 & 14.06 & 62.97 & 12.62 \\
\hline DV & 0.10 & 0.31 & 0.21 & 0.49 & 0.30 & 0.62 & 0.13 & 0.40 & 0.01 & 0.12 \\
\hline $\mathrm{ND}$ & 0.17 & 0.42 & 0.48 & 0.69 & 0.55 & 0.74 & 0.39 & 0.62 & 0.08 & 0.30 \\
\hline $\mathrm{SD}$ & 0.17 & 0.42 & 0.27 & 0.52 & 0.23 & 0.52 & 0.20 & 0.46 & 0.08 & 0.30 \\
\hline $\mathrm{VT}$ & 0.15 & 0.41 & 0.44 & 0.66 & 0.37 & 0.64 & 0.39 & 0.66 & 0.17 & 0.43 \\
\hline
\end{tabular}

Table 1. Observed and Modeled Buoy Variables

Variables were computed from the large-scale buoy array at Ice Station Weddell in 1992 with a 30 hour low-pass filter applied. Abbreviations are as follows: FD, free drift model; CAV, buoy results in cavitating fluid model; VP TRU, buoy results in the viscous-plastic model with truncated ellipse; VM, velocity magnitude in centimeters per second; DV, divergence; ND, normal deformation (elongation); SD, local shear; and VT, vorticity, all in units of $\times 10^{-6} \mathrm{~s}^{-1}$. 
of the predicted and observed velocities are close (free drift with no ocean current variance is about $114 \%$ of observed), predicted differential drift variances are substantially larger than observations, by $150 \%$ to $300 \%$ for free drift with no ocean current case. These results are similar to those found in the Beaufort Sea where the observed differential drift variances were found to be even smaller [Hibler et al., 1974].

Thus compared to the observed kinematic quantities in (1)-(3), we find that free drift considerations yield reasonable ice velocities but substantially overestimate differential drift motions. These results indicate that the ice interaction tends to maintain a more coherent structure to the adjacent ice floes than would occur if they were moving in free drift in response to wind and water forcing. Consequently, in the differential drift equations, it is clear that in order to explain the variance of the deformation, the internal ice stress term becomes more critical. We note that the ice stress does not need to be small in the drift equation case, only that the spatial derivatives of the ice stress may not always be large and that it is necessary to look at certain other characteristics of the ice drift statistics (as done by Hibler and Ip [1995]) to elucidate the role of ice interaction.

We will examine the response of both drift and differential drift under the assumption of different rheologies. Since we are numerically solving the nonlinear drift equations, we do not make the approximations used to obtain the above scaled differential drift equations but, instead, solve the full nonlinear drift equations and then compare statistics of the spatial derivatives of the predicted and observed drift.

\section{Numerical Experiment}

A numerical experiment was conducted to determine how well large-scale sea ice simulations perform compared with the ISW large-scale array observations. The ISW large-scale array is a unique data set to use for such a comparison, as both the drift and strain-rate components are statistically available from a collection of buoys. In addition, the six-point array spans $150 \mathrm{~km}$ by $100 \mathrm{~km}$. This scale is large enough to encompass six grid cells in a $50 \mathrm{~km}$ sea ice model which is a scale size that can be run to a full plastic solution in a reasonable amount of computer time. The ISW positions are located within the model grid every 3 hours between Julian days 63 and 150 in 1992. At these locations, velocities are extracted from the model's Cartesian grid and transformed spatially and with respect to vector orientation to an ISW grid oriented perpendicular and parallel to the shelf break, in $x$ and $y$ directions, respectively. These velocities, together with observed positions, are subjected to the deformation analysis described by Geiger et al. [1998]. An overview of the statistical functions is provided, for example, by Bendat and Piersol [1971], Hines and Montgomery [1990], and Geiger [1996]. An overview of the numerical scheme and rheologies is given in section 4.1 .

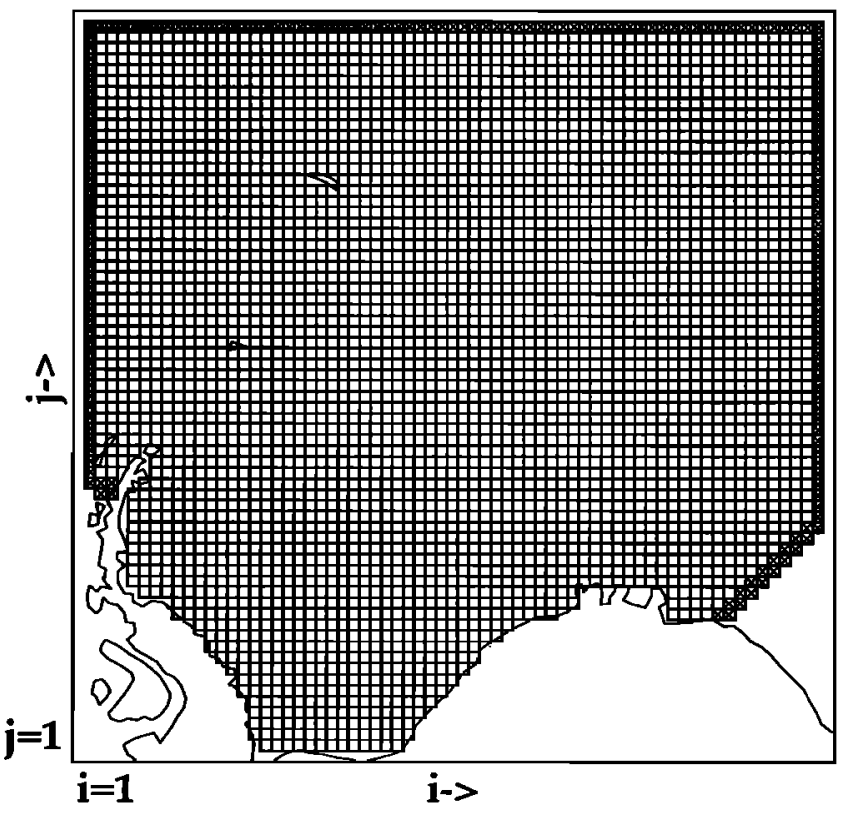

Figure 3. Numerical grid for regional study at 50 $\mathrm{km}$ resolution in Cartesian coordinates. Areas with no squares are land points, those with open squares are active ice points, and those with crossed squares are outflow points.

\subsection{Numerical Scheme}

Numerical simulations of sea ice were conducted for the Weddell Sea pack ice $\left(80^{\circ} \mathrm{S}\right.$ to $55^{\circ} \mathrm{S}$ latitude by $60^{\circ} \mathrm{W}$ to $20^{\circ} \mathrm{E}$ longitude) at a $50 \mathrm{~km}$ spatial resolution (see Figure 3) using the dynamic-thermodynamic sea ice model described by Zhang and Hibler [1997] for solving the viscous-plastic rheology and Flato and Hibler [1992] for the cavitating fluid rheology. The European Centre for Medium-Range Weather Forecasts (ECMWF) air temperature and pressure fields are used as input forcing at 6 hour intervals for the year 1992, while 1991 fields are used to spin up the model. Temporally constant ocean currents were generated from a composite for the Weddell Sea region based on geostrophic estimates of steric height anomaly contours by Olbers et al. [1992, Plate 56] from 1992 ISW current measurements and from subjective interpretation [Geiger, 1996]. A temporally varying, spatially constant mixed layer depth was generated using sinusoidal interpolation between $20 \mathrm{~m}$ on February 1 and $100 \mathrm{~m}$ on August 1 based on measurements from Gordon and Huber [1990]. For oceanic heat fluxes, estimates from ISW measurements [Lytle and Ackley, 1996] show about $7 \mathrm{~W} \mathrm{~m}^{-2}$ in the western Weddell Sea (about $60^{\circ} \mathrm{W}$ to $40^{\circ} \mathrm{W}$ ) in winter. From Gordon and Huber [1990], a winter heat flux value below the ice of about $37 \mathrm{~W} \mathrm{~m}^{-2}$ is estimated for the eastern Weddell (about $20^{\circ} \mathrm{W}$ to $10^{\circ} \mathrm{E}$ ). In summer, an average value of about $2 \mathrm{~W} \mathrm{~m}^{-2}$ for the entire region is typical [Parkinson and Washington, 1979]. Using these values, with linear interpolation between $40^{\circ} \mathrm{W}$ and $20^{\circ} \mathrm{W}$, we generated a longitudinal heat flux distribution. Local temporal variations between sum- 
mer and winter values were then used with the same sinusoidal interpolation method as for the mixed layer depth. Relative humidity is based on climatological 30 year monthly mean surface dew point and air temperature from Taljaard et al. [1969].

To make a more precise comparison to observations, the numerical code was set up to approach the full plastic solution by means of a pseudo time stepping procedure performed at every 3 hour physical time step (see Appendix B). To do this, the momentum balance calculations described by Zhang and Hibler [1997] were iteratively computed 10 times to produce 10 pseudo time steps. In this way, the velocity converges in the momentum balance based on the computed pressure and viscosity terms (as accomplished with one pseudo time step), but then, with each successive pseudo time step, the pressure and viscosity terms are updated by the new velocities such that velocity, pressure stress, and bulk and shear viscosities all approach mutually convergent solutions (i.e., a plastic solution). This method adds to the computational time of the model; the compromise of 10 pseudo time steps is judiciously selected to provide reasonable plastic solutions within a reasonable amount of computer time. Comparisons, discussed in section 5 (see Figure 9), illustrate how well 10 pseudo time steps work as seen by the straight clustering of data on the yield curve. With fewer pseudo time steps, there is a greater spread of data normal to the yield curve.

The models are run with eight time steps a day (3 hour intervals) and with $50 \mathrm{~km}$ resolution to match the Argos sampling rate in time and space, respectively. The times and positions of the buoys in the model are those of the observed ISW buoy sites, with velocity extracted from the model. These velocities are then processed in the same manner as the observed buoy velocities [Geiger et al., 1998] to obtain the centroid velocity and local deformation results from the model. Since deformation is essentially a measure of the spatial variability of the velocity field, this method of extracting data from the model provides a reasonable estimate of the model deformation results compared with observations.

\subsection{Ice Rheologies}

Three basic rheology types are used for this experiment: the viscous-plastic elliptical yield curve with energy-conserving modifications of Hibler and Ip [1995] and modifications to remove tensile stress [Hibler and Schulson, 1997], free drift (viscous-plastic code with maximum pressure equal to zero), and cavitating fluid [Flato and Hibler, 1992]. The viscous-plastic rheology is the most physically realistic of the three rheologies studied and so, in addition to the free drift and cavitating fluid rheologies, the viscous-plastic rheology is examined with a number of modifications. These modifications are best explained in an invariant principal axis space $\left(\sigma_{1}, \sigma_{2}\right)$ for two dimensions, where $\sigma_{\mathrm{I}}$ is divergence, $\sigma_{\mathrm{II}}$ is maximum shear, $\sigma_{1}=\sigma_{\mathrm{I}}+\sigma_{\mathrm{II}}$ and
$\sigma_{2}=\sigma_{\mathrm{I}}-\sigma_{\mathrm{II}}$ (for more details, see, for example, Geiger et al. [1998]). Using Figure 4 as a guide, we find that the fundamental difference between the viscous-plastic model and cavitating fluid is that the cavitating fluid is a material that resists against applied pressure (compressive stresses) but not against shear stress (Figure 4b). The viscous plastic, on the other hand, is a rheology type that reacts to both compressive and shear stresses with a response, prior to failure, of viscous creep (shaded region in Figure 4) and a plastic deformation behavior upon yielding (bold outline of ellipses in Figure 4).

A number of descriptions of the viscous behavior have been developed within the plastic elliptical yield curve framework. The original use of the viscous-plastic rheology in sea ice was presented by Hibler [1979], with regions of similar viscous flow oriented as concentric ellipses within the plastic yield ellipse (the so-called concentric ellipse yield curve; Figure 4c). One drawback to this configuration is that the field will experience creep flow even in the absence of forcing. Further development of this rheology by Hibler and Ip [1995] was made to eliminate this problem by adjusting the maximum pressure at each point and time of the momentum balance (see Appendix C). In this way, lines of constant viscosity pass through the origin of the stress field such that no creep exists when no external forces are present, a physically realistic result. This addition (Figure 4d) has been described as the replacement method [Hibler and Ip, 1995] because of the replacement of the constant maximum pressure with a pressure in creep state.

An additional development to the rheology is the elimination of tensile stress to produce a truncated ellipse (Figure 4e), with constant viscous states anchored at the origin and no stress states possible in the positive domains of the principal axes (see details in Appendix C). Not only is this addition physically realistic at the geophysical scale, but it is also analogous to the rheological configuration found from three-dimensional stress tests in laboratory experiments at the meter scale [ $\mathrm{Hi}$ bler and Schulson, 1997], making this a practical rheology candidate for multiscale studies of sea ice rheology.

\section{Comparison Results}

Results from the numerical experiments described in section 4 indicate that overall, the viscous-plastic model performs the best out of the three rheologies of free drift, cavitating fluid, and viscous plastic with truncated ellipse. Because of this, we present our results in two parts. First, we present an interrheology comparison to show how the three rheologies perform compared with the observed array. Second, we conduct an additional set of comparisons based on modifications of the viscous-plastic rheology.

\subsection{Interrheology Comparison}

Using the viscous-plastic rheology with a truncated ellipse closure scheme as an example in Figure 5, we find 

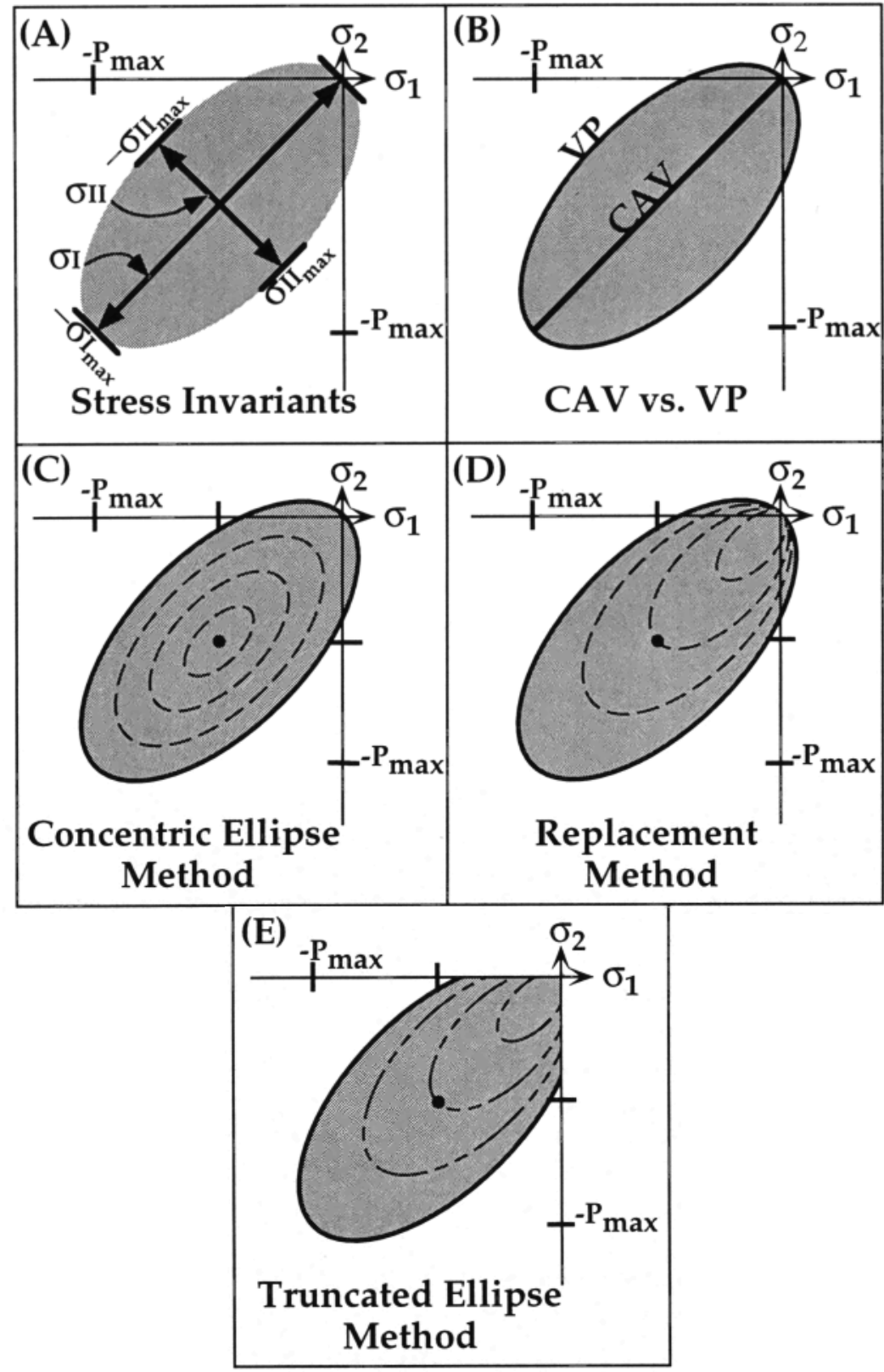

Figure 4. Description of the invariant principal stress components $\left(\sigma_{1}, \sigma_{2}, \sigma_{\mathrm{I}}\right.$, and $\left.\sigma_{\mathrm{II}}\right)$ and sea ice rheologies in two-dimensional principal axis space for (a) stress invariants, (b) cavitating fluid versus viscous-plastic rheologies, (c) concentric ellipse method, (d) replacement method, and truncated ellipse method. $P_{\max }$ is a positive pressure term, and $P_{\max }$ and all $\sigma$ values are in Pascals per meter. CAV represents the cavitating fluid rheology, and VP is the viscous-plastic rheology with its closure methods (see text for details).

that the low-frequency signals are well reproduced by the models while higher frequencies, specifically those of 1 day or less, are not. Despite the presence of a 3 hour time step, an inertial term in the model momentum balance and subdaily atmospheric wind forcing, the powers of the daily and subdaily frequencies are modeled far below those observed. As seen in the comparison to observations (Figure 2), the models lack the daily and subdaily tidal and inertial oscillation processes. While inclusion of tidal forcing would have an impact, construction of the air-ice-sea boundary layer formulation in the current ice model assumes steady motion over several inertial periods, resulting in a large damping of subdaily frequencies in the model. Indeed, mechanistic model studies [Hibler et al., 1998] employing less damped boundary formulations demonstrate that the interaction of kinematic waves and inertial motion can lead to substantial inertial power in ice deformation. Because of such considerations, we expect comparisons between modeled and observed velocities to be realistic here only for timescales greater than 1 day.

Frequency comparisons between modeled and observed results using a 30 hour low-pass filter retain most of the velocity signal from both the models and observations. 

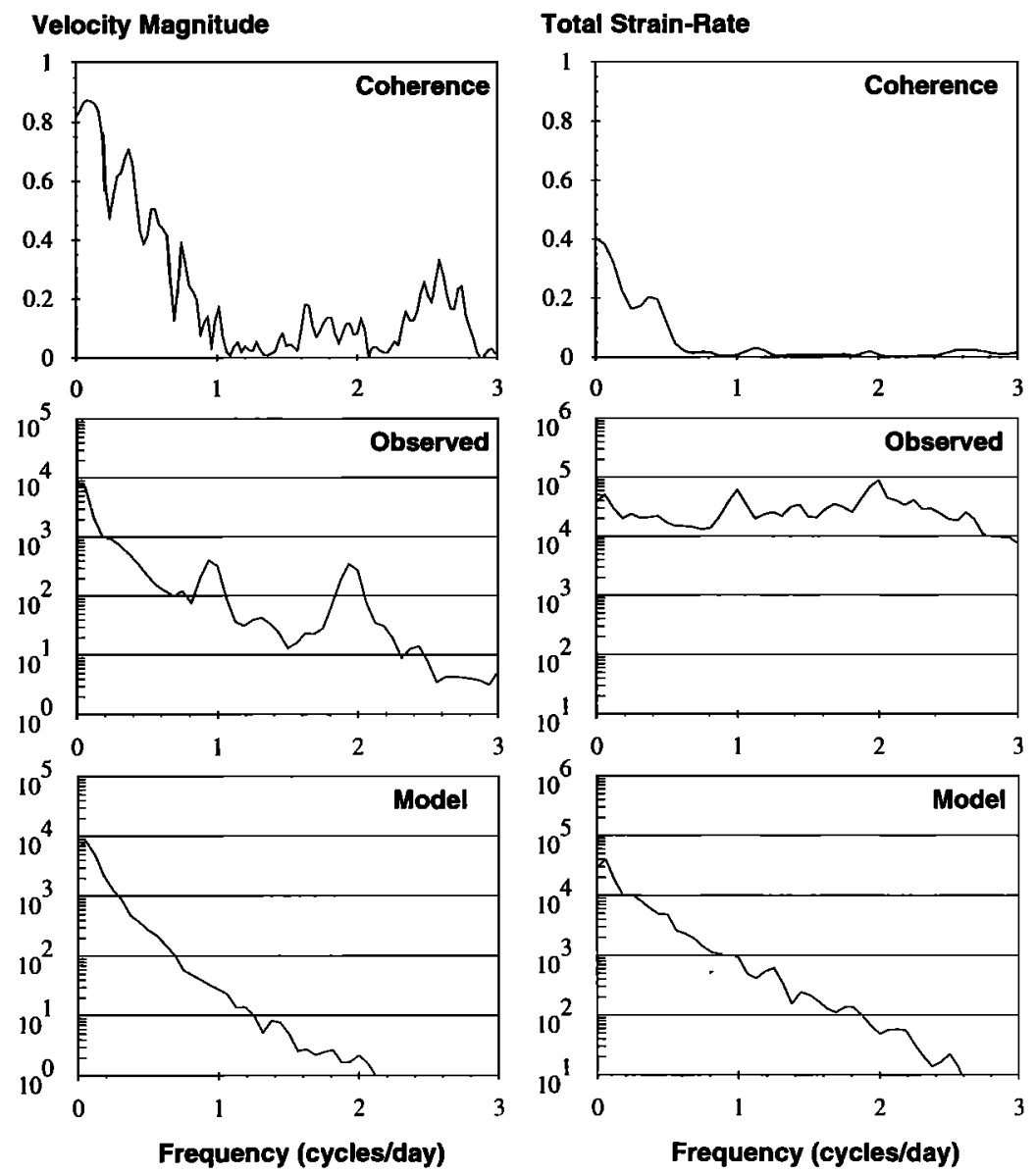

Figure 5. (Top) normalized cross-spectral coherence for (left) power of ice velocity and (right) power of total strain rate for spectra from (middle) ISW observations versus (bottom) a viscous plastic with truncated ellipse sea ice model. Power spectra are presented in power density form, with velocity in square meters per second and strain rate in units of $\times 10^{-12} \mathrm{~s}^{-1}$.

Running a 30 hour low-pass filter (LPF) over the model has little effect on the frequency domain results (or any of the statistical results examined) but is applied to the models for consistency.

Time series plots of velocity magnitude and the two invariant deformation components of divergence and maximum shear for each of the three models are shown in Figure 6, together with the same information for the 30 hour LPF case of the observations. Since the maximum shear is usually chosen as a positive quantity (see Appendix A), its two components of local shear and normal deformation are also included in Figure 6. Qualitatively, the modeled velocity corresponds well to observed velocity for all modeled cases, with specific highvelocity events (peaks on the plots) coinciding in time very closely. The deformation components, on the other hand, are not as compatible. While the model deformation values scale comparably to those observed, they do a poor job at matching specific time episodes, although there are some exceptions, particularly for the maximum shear component (shown in Figure 6 with plus and minus values, either of which is possible at any point in time; see (A3)). A mean close to zero in the observed divergence (DV), normal deformation/elongation (ND), and local shear (SD) are reproduced in the viscousplastic case but not in the free drift (FD) or cavitating fluid (CAV) cases, which exhibit a number of strong low-frequency signals from 5 to 60 day periods, resulting in strong convergence (defined as positive divergence here) of the order of $10^{-6} \approx 10 \%$ per day from about day 90 to 120 . These strong convergence episodes are not seen in the observations and relate to physically unrealistic instances of piling up of ice locally. While the cavitating fluid case is not as extreme as the free drift case, the cavitating fluid, with its ability to resist compressive stress, is providing only part of the answer needed to compute deformation correctly.

Inclusion of shear stress, as in the case of the viscousplastic (VP) rheology, seems to be necessary in the model. Of all the deformation components observed, divergence is the most poorly reproduced while maximum shear is the best. For the latter, 30 hour LPF deformation results using the viscous-plastic model are remarkably similar to those observed, including the re- 
(a.) Free Drift
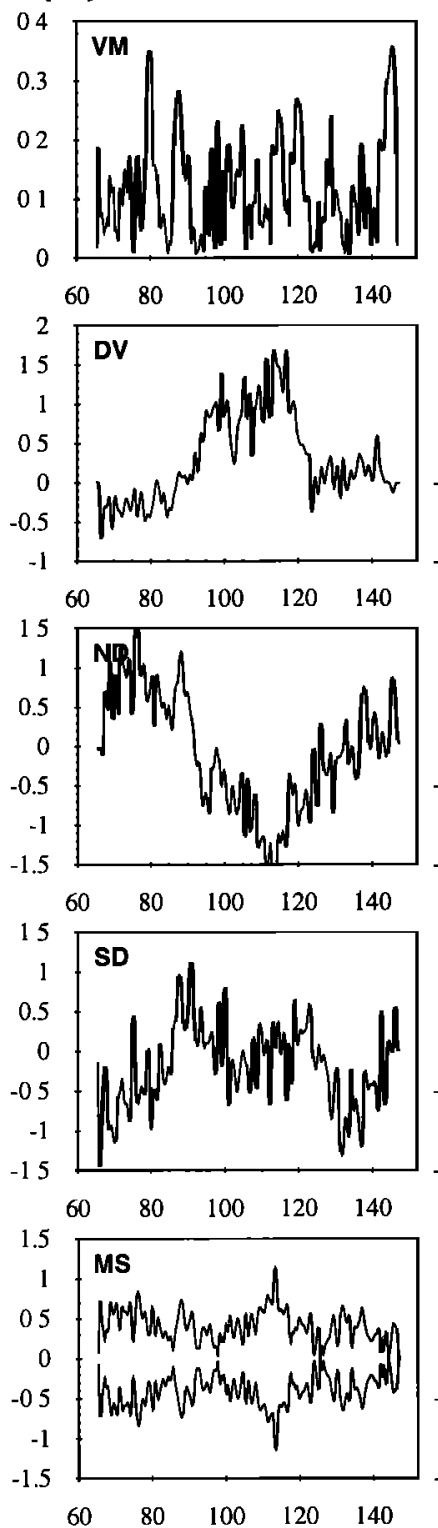

Julian Days (b.) Cavitating Fluid

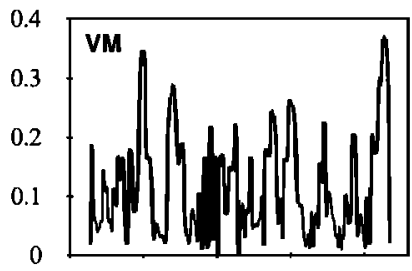

$\begin{array}{lllll}60 & 80 & 100 & 120 & 140\end{array}$
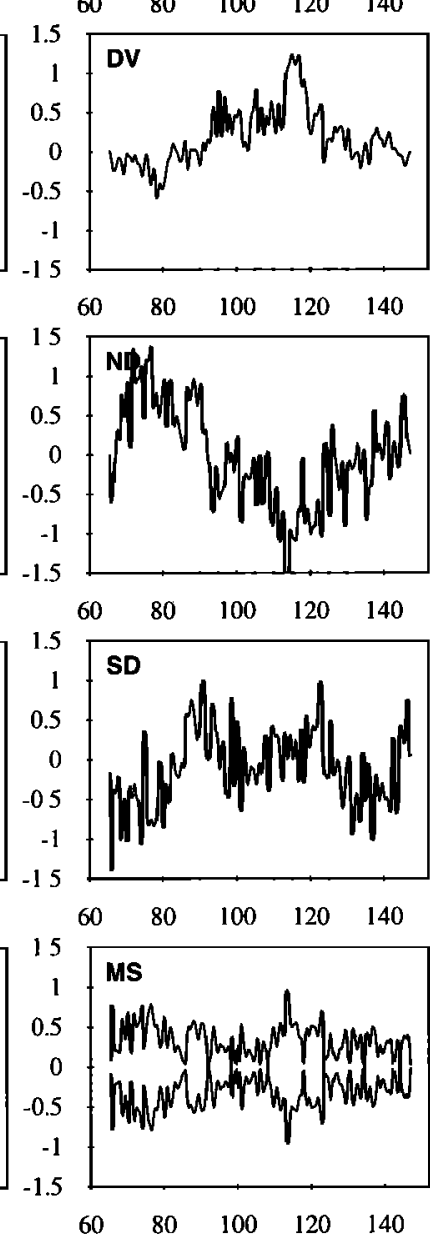

Julian Days (c.) Viscous-Plastic
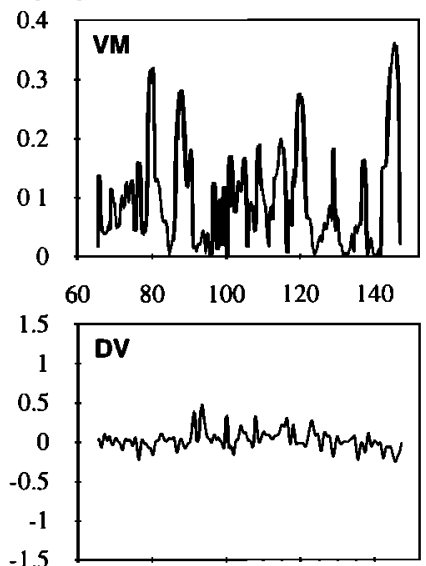

$\begin{array}{lllll}60 & 80 & 100 & 120 & 140\end{array}$

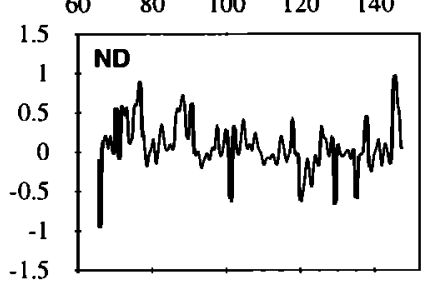

$\begin{array}{lllll}60 & 80 & 100 & 120 & 140\end{array}$
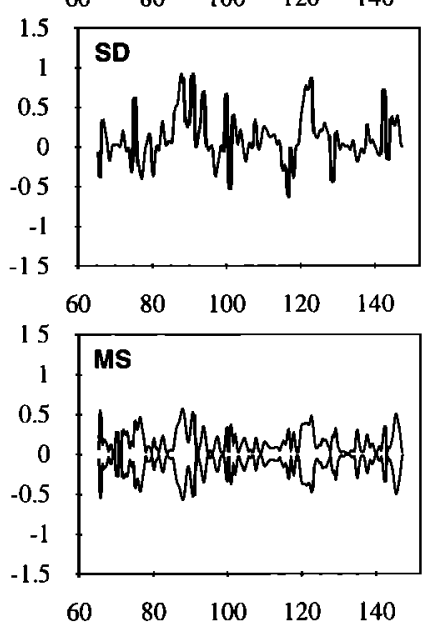

Julian Days (d.) ISW-Observed

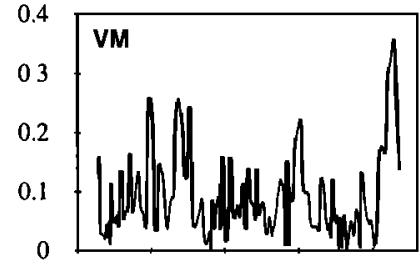

$\begin{array}{lllll}60 & 80 & 100 & 120 & 140\end{array}$

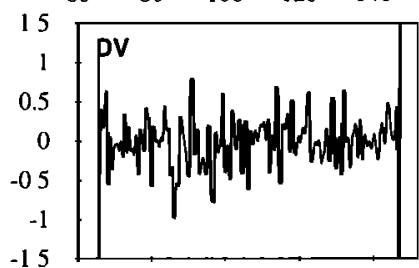

$\begin{array}{lllll}60 & 80 & 100 & 120 & 140\end{array}$

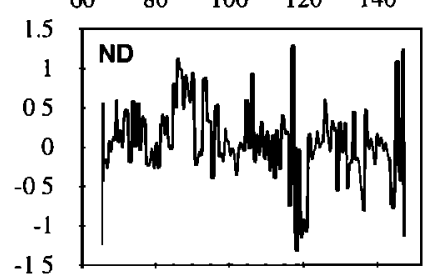

$\begin{array}{lllll}60 & 80 & 100 & 120 & 140\end{array}$
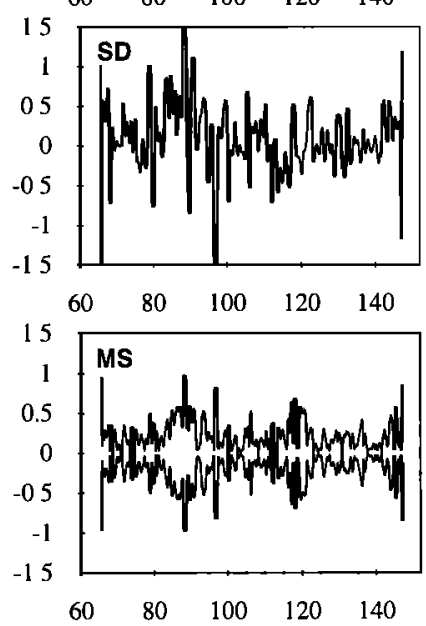

Julian Days

Figure 6. Time series of buoy array centroid for variables of velocity magnitude (VM), divergence (DV), normal deformation (ND), local shear deformation (SD), and maximum shear (MS) as computed from multiple linear regression analysis using 30 hour low-pass filter for (a) free drift, (b) cavitating fluid, and (c) viscous-plastic models versus (d) ISW observations. Velocities are in meters per second, and deformation terms are in units of $\times 10^{-6} \mathrm{~s}^{-1}$ for the three model cases and the observed ISW array. Since maximum shear is computed from a square root (see (A3)), both positive and negative roots are plotted such that any value at any point in the time series could be either positive or negative.

production of peaks and troughs at the same time as those observed, albeit with significantly smaller magnitudes.

While the velocities look similar in the time series plots, slight differences in velocity result in both major differences in space, as seen in deformation, and in time. To illustrate this, progressive vector plots of velocity for the observed, free drift, cavitating fluid, and viscous plastic with truncated ellipse are shown in Figure 7. Comparing observations to FD in Figure 7, we see that FD predicts faster northward motion in the west than east, as also seen in the observations, but the drift pattern is distorted, especially at the four eastern sites. This distortion produces the poor deformation outcome seen in the time series plots. The cavitating fluid model compares somewhat better than FD to the 


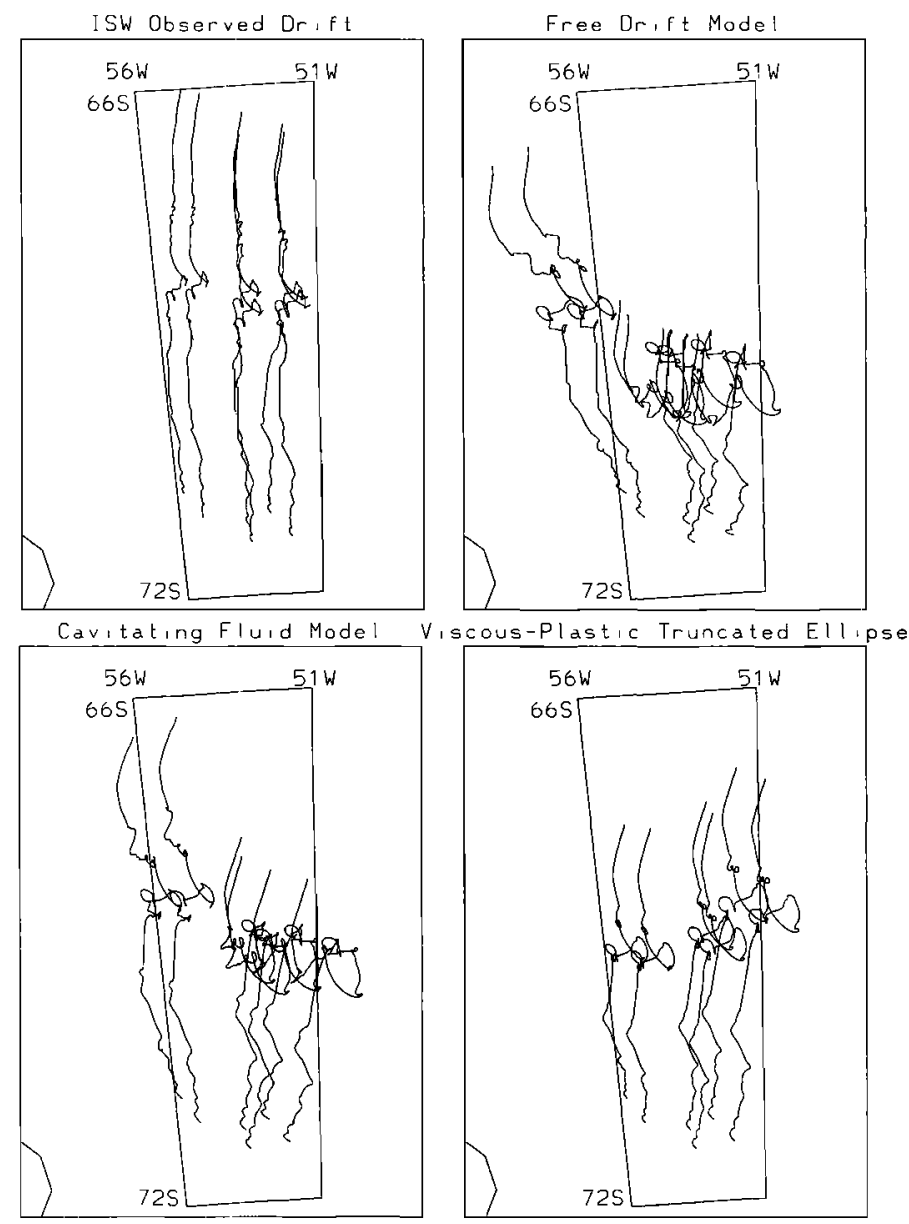

Figure 7. Progressive vector plots of buoys as derived from observations and three simulation results. Vectors are computed from 3 hour sampling of velocities subjected to a 30 hour low-pass filter.

observations with the two western buoys drifting similar to that seen in the observations. The four eastern sites, however, are still poorly reproduced.

The viscous-plastic model with truncated ellipse in Figure 7 shows many of the short-term features in the observations and a trajectory that is, overall, the closest to observations. However, particulars of the drift are distorted, specifically the intermittent drift perturbations (wiggles in the observed 30 hour LPF drift) and the observed strong west to east shear in the net northward drift that is reversed in the TRU model. In an attempt to see if the drag coefficient might be responsible for the stronger northward flow in the west, the drag coefficients were modified according to Fischer and Lemke [1994] to include a reduced ocean $\operatorname{drag}\left(C_{w}^{*}=0.0030\right.$, down from $C_{w}^{*}=0.0055 \mathrm{~kg} \mathrm{~m}^{-2} \mathrm{~s}^{-1}$ ) and increased wind $\operatorname{drag}\left(C_{a}^{*}=0.0015\right.$, up from $\left.C_{a}^{*}=0.0012 \mathrm{~kg} \mathrm{~m}^{-2} \mathrm{~s}^{-1}\right)$. Unfortunately (not shown here), this leads to an overall northward flow that greatly exceeds observations and proportionally increases the east to west shear, with the eastern buoys still moving too fast northward relative to the western buoys.

Overall assessment using progressive vector plots shows that none of these models properly reproduces all the features observed, but the cavitating fluid best reproduces the general western intensification flow seen in the observations while the viscous-plastic model (due to inclusion of shear) spatially drifts similar to observations, except for the western intensification. This is occurring despite the fact that a western intensification ocean current is added in the models as prescribed by the observations [Geiger, 1996].

Quantitatively, with a 30 hour low-pass filter, a crossspectral coherence close to $90 \%$ was found for $\mathrm{FD}, \mathrm{CAV}$, and VP models versus observed buoy velocities (see Appendix A). From the example shown in Figure 5, we see that this is attributed to the high peak of coherence around the 5 to 8 day period (close to that of storm activity) and is believed to be a result of the ECMWF input atmospheric pressure fields. Conversely, coherence between the model and observed total strain rates are much lower (highest value is for the viscous-plastic rheology at $40 \%$ coherence as seen in Figure 5). Normalized cross correlation results as shown in Figure 8 additionally exhibit nearly the same result, with modeled velocity comparing better than $80 \%$ to observed in all but direction, which correlates poorly $(<50 \%)$. Deformation variables correlate less well (e.g., the best result 


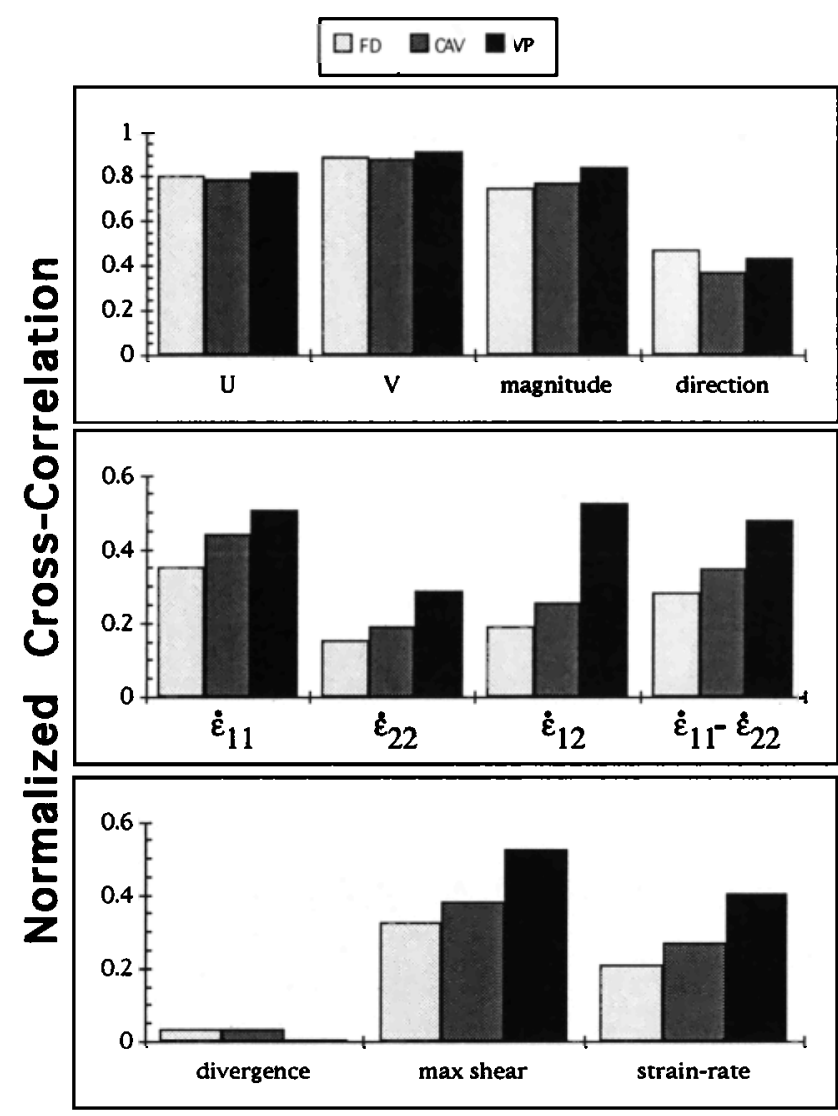

Categories

Figure 8. Normalized cross correlation coefficients of drift $u, v$ and deformation components $\dot{\epsilon}_{i j}$ between observations and sea ice models of free drift (FD), cavitating fluid (CAV), and viscous-plastic (VP) rheologies.

is for maximum shear at about $50 \%$ for the viscousplastic case). However, for both invariant and noninvariant strain-rate components (Figure 8 ), the different rheologies show a clear distinction in correlation versus those observed (20\% for FD, $28 \%$ for CAV, $40 \%$ for VP total strain rates). Additionally, in all cases, maximum shear (see Appendix A) is modeled much better than divergence which poorly correlates in this study for all three rheology types tested. The similarity in velocity coherence, despite the different rheological types, is attributed to high quality in the ECMWF 6 hour interval atmospheric pressure fields used to drive the models. The low correlated velocity direction is apparently a typical problem associated with the input fields in this region (P. Lemke, personal communication, 1997) and is a likely contributor to the low divergence values in all the model results.

Overall, from this interrheology comparison, we find that first, the velocity is reproduced well, no matter which rheology is chosen and is primarily dependent on the quality of the wind velocity field. Hence, to within about $80 \%$ to $90 \%$ confidence, the ice velocity responds primarily to wind and water forcing. Second, the deformation of the field is more difficult to predict in the models, at most, only $50 \%$ of the coherence attainable in this study. A noticeable improvement is found in model deformation for the more realistic rheologies, which include both compressive and shear stresses. Finally, the strain rate, and not the velocity, is found to be the more critical parameter to compare in model results in terms of testing a constitutive relation.

\subsection{Comparison of Viscous-Plastic Modifications}

One conclusion from the results described in section 5.1 is that inclusion of both divergent and shear deformation produces the best rheological results when compared with observations. The next question is, which components of the best model could be modified to improve it even further? The aim of this section is to address this question by examining the response of some recently suggested modifications to the viscous-plastic model in order to see how well they compare with the observed results. As discussed in section 1, the viscousplastic closure scheme has recently gone through a number of modifications. First, the original concentric ellipse method [Hibler, 1979] has been modified to a truncated ellipse [Hibler and Schulson, 1997], which is developed from the replacement method of Hibler and Ip [1995]. Second, in terms of the mechanical behavior, a more brittle or ductile response can be achieved by increasing or decreasing, respectively, the bulk viscosity term $\zeta_{\max }$. For purposes of simplicity, let us consider the case of increased ductility $\left(\zeta_{\max } \longrightarrow \zeta_{\max } / 100\right)$ versus the more brittle standard case. Third, in the literature, Fischer and Lemke [1994] found good correlation in buoy drift statistics for the Weddell Sea by modifying the wind and water drag coefficients from those of Hibler [1979] from $0.0012,0.0055 \mathrm{~kg} \mathrm{~m}^{-2} \mathrm{~s}^{-1}$ to 0.0015 , $0.0030 \mathrm{~kg} \mathrm{~m}^{-2} \mathrm{~s}^{-1}$, respectively. Since the air drag is only slightly different while the water drag is significantly reduced, let us call this a reduced water drag case. Finally, an important issue in numerical modeling is scale and how it impacts a model's results. In this case, let us consider a $25 \mathrm{~km}$ model (twice the standard resolution). With the above modifications, we cover some of the current viscous-plastic rheology sensitivity categories being examined today. Since the truncated ellipse is more realistic than the concentric ellipse, the former will be the standard case, with other case studies as a modification of that model. Therefore, in addition to free drift and cavitating fluid, we add the original viscous-plastic model with concentric ellipse (CON), our identified standard viscous-plastic model with truncated ellipse (TRU), a ductile case (DUCT = TRU with $\left.\zeta_{\max } \longrightarrow \zeta_{\max } / 100\right)$, the reduced water drag case (DRAG) described above, and a $25 \mathrm{~km}$ version of TRU.

Differences in time series, frequency domain, and probability distribution are notable between the various viscous-plastic cases, but their differences are best 

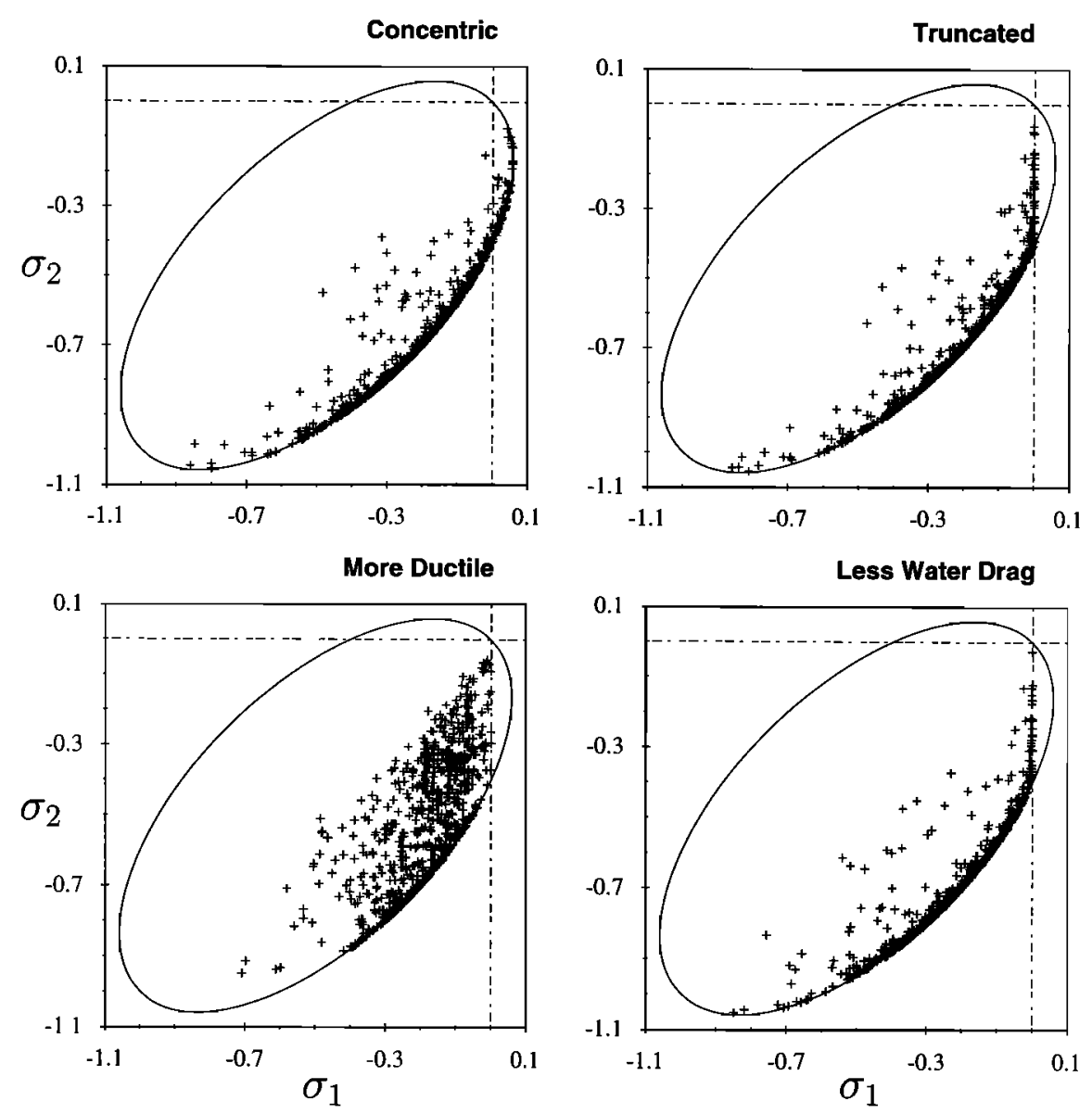

Figure 9. Scatterplots in normalized principal axis stress space $\left(\sigma_{1}, \sigma_{2}\right)$ for 3 hour interval ISW centroid locations in the viscous-plastic models with concentric ellipse closure, truncated ellipse closure, a ductile state of truncated with $\zeta_{\max } \rightarrow \zeta_{\max } / 100$ (more ductile), and a reduced water drag case (see text for details).

exemplified in principal axis space. Three hour interval samples of the buoy array centroid are shown in Figure 9 in principal axis space $\left(\sigma_{1}, \sigma_{2}\right)$ for the different viscous-plastic cases chosen. The primary difference between the concentric and truncated closure scheme occurs near the origin where the concentric ellipse extends into positive $\sigma_{1}$ and $\sigma_{2}$ quadrants and thus experiences tensile stress. On a large scale, this is physically unrealistic. The truncated case, on the other hand, has no tensile stress and, furthermore, owing to the replacement method, has fewer points close to the center of the ellipse at $-P_{\max } / 2$ and a slightly different distribution of points along the plastic yield limit. When a more ductile state is employed (Figure 9, bottom), a considerably greater number of points are located in the viscous region, with far fewer points extending into the bottom of the yield curve (i.e., fewer large negative values, less convergent). The reduced water drag case looks very similar to the standard (TRU) run, except for a few more points in the interior in the viscous region, and is slightly more convergent, but this is fairly subtle. The $25 \mathrm{~km}$ case has similar subtle differences in principal axis space (not shown here) versus the $50 \mathrm{~km}$ standard case, with only slight differences in both drift and deformation (Figure 10). The biggest improvement in the increased resolution case is an increase in coherence from $<20 \%$ to nearly $40 \%$ at daily and $\approx 17$ hour periods, neither of which relates to the inertial period (which is $\approx 12$ hours in this region). This increased coherence has only subtle effects in the divergence and strain-rate components, as exemplified in Figure 10.

In all of the cases shown in Figure 9, the stresses are concentrated near $\sigma_{\mathrm{II} \max }$, the maximum point of the maximum shear in principal axis space (see Figure 4a). There are only a few points approaching the maximum convergence point $-\sigma_{I_{\max }}$. Recalling the time series in Figure 6, this concurs with the smaller model divergence values versus those observed. From both the observed strain rate and the modeled stress information above, we hypothesize that the observed stress state is similar to the ones shown in Figure 9 but with the main concentration of points located on the yield curve between $-\sigma_{I_{\max }}$ and $\sigma_{I_{\max }}$ rather than clustered about $\sigma_{\mathrm{II} \max }$. Hence none of these modifications produces the divergence desired. The ductile case works against increasing the convergence, while the modified water drag 

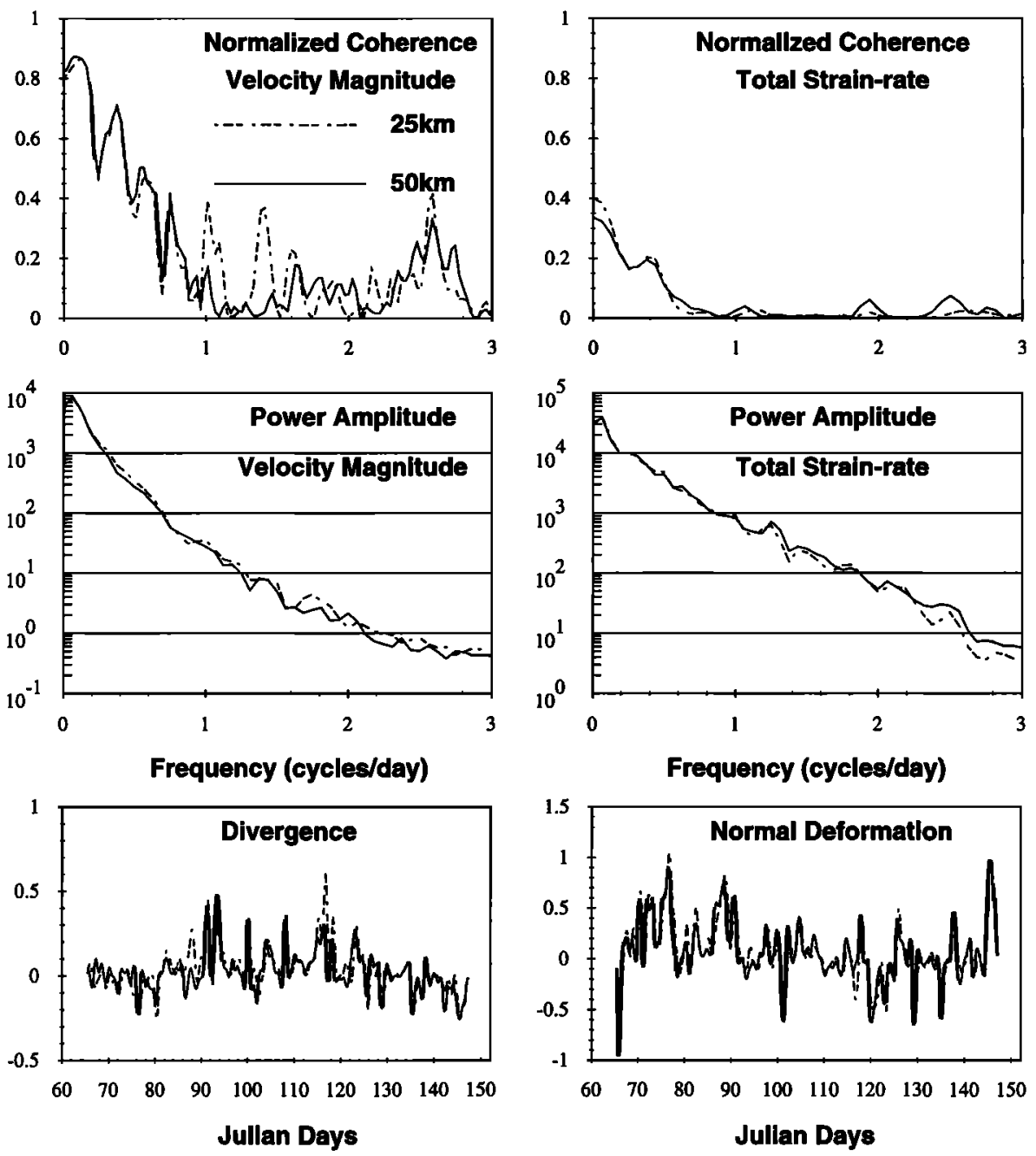

Figure 10. Examples of results from a $25 \mathrm{~km}$ run of the viscous-plastic rheology with truncated ellipse versus the same model at $50 \mathrm{~km}$. Power density of velocity is in square meters per second, and divergence time series is in units of $\times 10^{-6} \mathrm{~s}^{-1}$.

has a few more convergent points than the truncated and concentric ellipse cases and hence comes closer to the observed divergence, but only subtly so. Increasing the ductility only increases the number of points on the yield curve but does little to move them into the more convergent region of the curve.

No observed stresses are available, but the observed strain rates are computed and can be compared directly with the modeled strain rates. The performance of the modeled strain rates versus observed is seen in the scatterplots in Figure 11 for the cavitating fluid, truncated ellipse at 50 and $25 \mathrm{~km}$, and the reduced water drag cases. The cavitating fluid (Figure 11a) and, similarly, the free drift case (not shown) reproduce velocity that is slightly more scattered than for the viscous-plastic case (Figure 11b), while modeled deformation variability in the cavitating fluid is far greater than that observed (i.e., lots of scatter in the model direction versus little scatter in the observed direction). For the viscousplastic model (Figure 11b), a much closer one-to-one relationship is established between model and observa- tions for the shear terms while the model clearly underestimates the divergence versus that observed. The compressive and shear deformation terms, which are the components of the maximum shear (see (4), (5), and (A3)), are used in place of the maximum shear to eliminate dealing with the sign discrepancy connected with the square root of the maximum shear (see Appendix A). Both the compressive and shear deformations are aligned along the diagonal but are still narrower in the vertical (model) direction than in the observations, indicating that the model is underpredicting both of these quantities. By increasing resolution to $25 \mathrm{~km}$ for the viscous-plastic model (Figure 11c), the results change only slightly, with the most improvement in increasing the model variability in the elongation and local shear. The modified drag case overpredicts velocity relative to observations (Figure 11d) but increases the model variability for divergence and shear terms to more closely resemble those observed.

To get a more quantitative sense of this variability, Table 1 shows the temporal variance and rms of the 
(a.) Cav

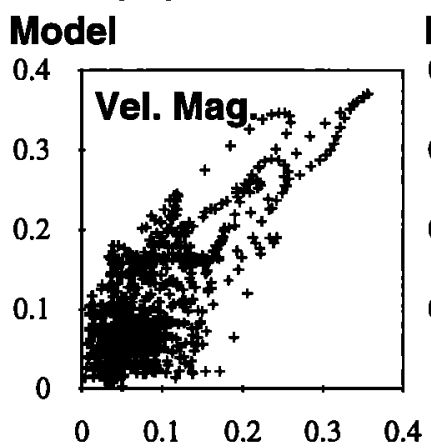

(b.) Tru

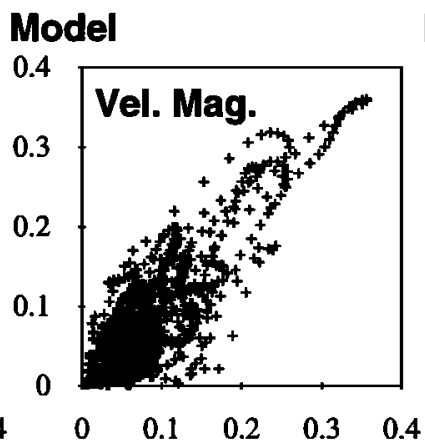

(c.) $25 \mathrm{~km}$ Tru

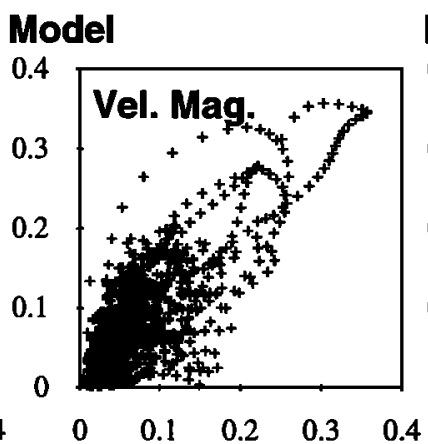

(d.) Drag

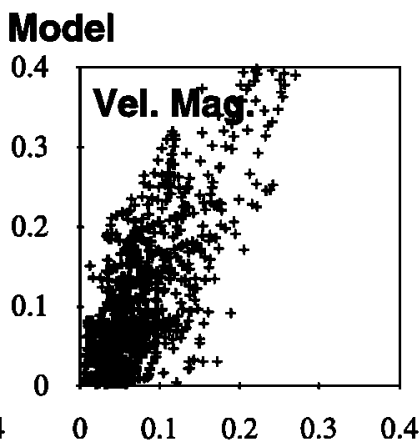

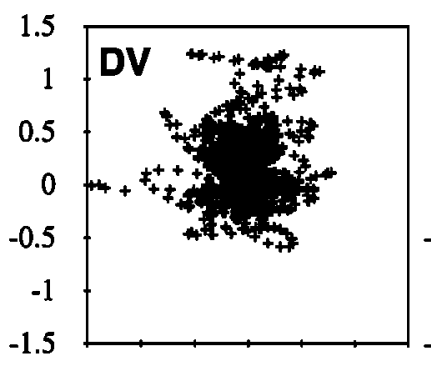
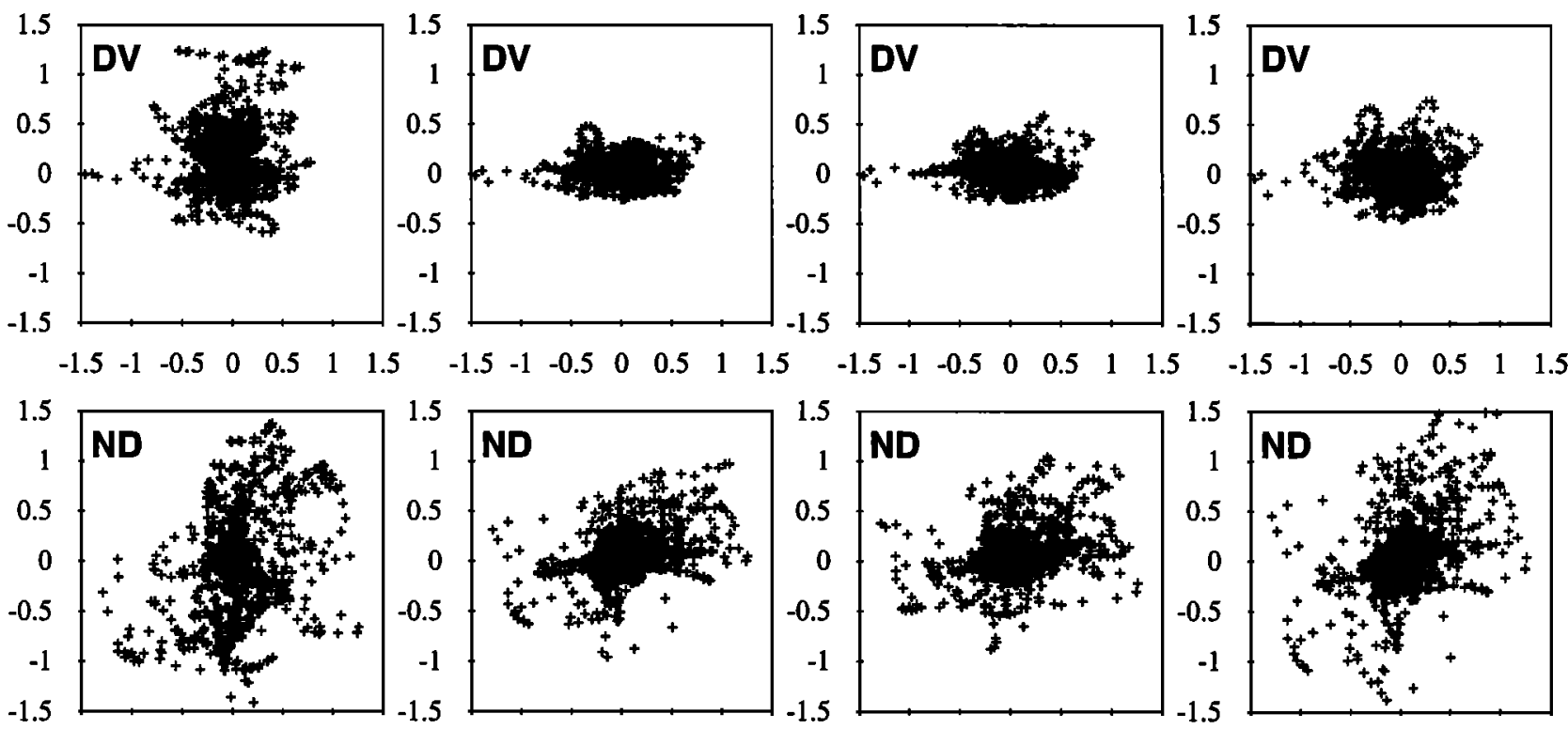

$\begin{array}{lllllll}-1.5 & -1 & -0.5 & 0 & 0.5 & 1 & 1.5\end{array}$

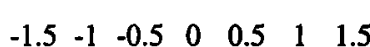

$\begin{array}{lllllll}-1.5 & -1 & -0.5 & 0 & 0.5 & 1 & 1.5\end{array}$
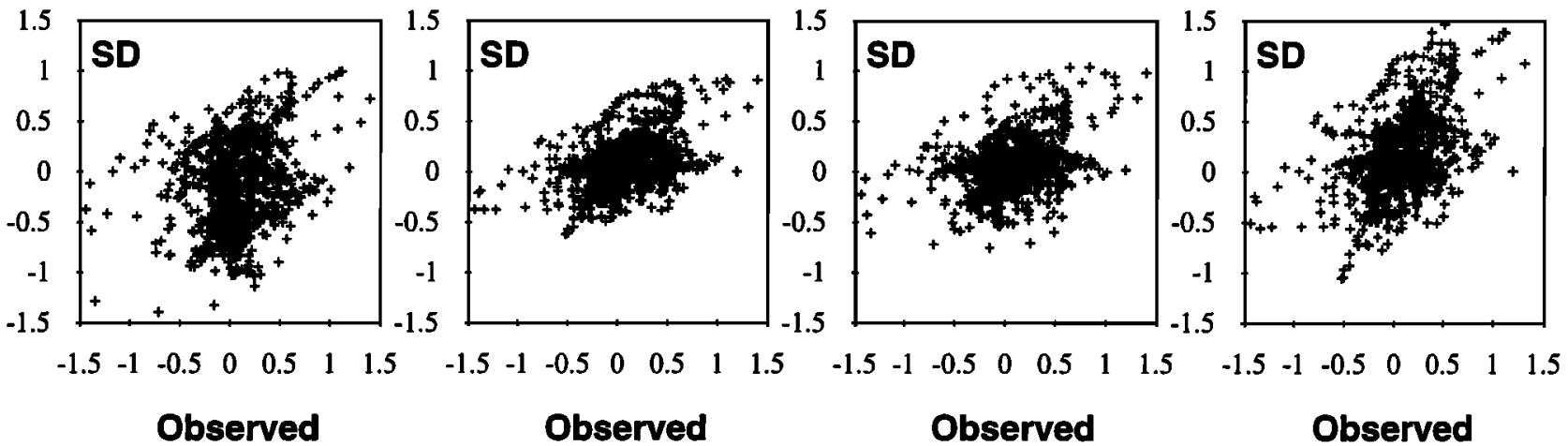

Figure 11. Scatterplots of correlation between 3 hour interval ISW centroid observations against (a) $50 \mathrm{~km}$ cavitating fluid, (b) $50 \mathrm{~km}$ viscous plastic with truncated ellipse, (c) $25 \mathrm{~km}$ version of the truncated ellipse, and (d) $50 \mathrm{~km}$ truncated case with modified drag. Velocity magnitude is in meters per second, and selected deformation terms are in units of $\times 10^{-6} \mathrm{~s}^{-1}$ for divergence (DV), normal deformation/local elongation (ND), and local shear (SD).

observed and simulated buoy array variables. The rms variability in the velocity magnitude decreases progressively for free drift, then cavitating fluid, and finally the viscous-plastic case with truncated ellipse (TRU), with TRU being the closest to that observed (11.92 for observations, 12.62 for TRU). The observed velocity results differ only slightly from the model examples in Table 1 (23\% for free drift with ocean current and $30 \%$ for TRU for variance relative error between models and observations). However, we note a clear distinction in results of the different rheologies for kinematic quantities. Free drift with ocean current has substantially greater variance than observations, e.g., relative errors for divergence, elongation, local shear, and vorticity are $200 \%, 224 \%, 35 \%$, and $59 \%$, respectively. However, with the addition of ice interaction, the variance and 
rms decrease in the deformation components to $90 \%$, $53 \%, 52 \%$, and $13 \%$ for viscous plastic, respectively. In particular, the divergence and components of maximum shear (ND and SD) have an observed variance and rms which lies between that of the cavitiating fluid model and the viscous-plastic model. Hence the results from Table 1 and the time series from Figure 6 indicate the following. Shear is a needed component for moderating the long low-frequency 5 to 60 day unobserved high variability in free drift and cavitating fluid. However, the current viscous-plastic simulation seems to dampen the strain rate field too much, particularly the divergence, producing a variance that is too low. This result suggests that a physical process designed to increase variability and divergence in the viscous-plastic model should improve model results for this region. One possible consideration is the inclusion of subdaily processes where inertial oscillations, working either in conjunction with or as a result of tidal motion, are allowed to evolve without being overdamped.

To better understand the kinematic and deformation components, a representative plot of the probability density distribution and resultant $\chi^{2}$ distribution is shown in Figure 12. The $\partial v / \partial y$ component is shown for the observed buoy array and those simulated in the following four cases: the free drift, cavitating fluid, viscous plastic with truncated ellipse (TRU), and TRU with a reduced water drag. From this example, one result that stands out is that the probability distribution is basically Gaussian about a zero mean. This distribution is typical of all the observed deformation and kinematic components examined Geiger et al. [1996]. In the absence of modeled shear stress, the free drift and cavitating fluid models do not reproduce this distribution and, instead, tend to be more divergent than observed. The viscous-plastic cases, however, are more Gaussian in their deformation distributions, with the biggest difference in probability density near the mean. Chi-square distribution further indicates specific regions within the probability distribution where the models overpredict or underpredict the observed. While none of the viscousplastic modifications compared overall better than the observations, their close correspondance to the observed probability distribution in a number of the components indicates that the viscous-plastic solution is at least on the right track to a correct solution.

The overall $\chi^{2}$ statistics from the probability distribution function are summarized in Table 2 . Looking first at the categories for velocity, the best $\chi^{2}$ (lowest value) is for the reduced ductility case because of its ability to match observed smaller velocity magnitudes where ductile creep is most effective. This is followed by the $25 \mathrm{~km}$ model, with the worse two cases being the reduced water drag and concentric ellipse cases, which are even worse than for the free drift and cavitating fluid models. For velocity direction, free drift and cavitating fluid have the highest $\chi^{2}$, with concentric and truncated ellipses producing the best result. In considering both magnitude and direction results, free drift, cavitating fluid, and reduced drag have the least favorable results while the remaining four appear indistinguishably close.

In terms of individual deformation components ( $\mathrm{Ta}-$ ble 2), things become a bit more distinct. First, the free drift and cavitating fluid results are clearly the worst. The case of reduced drag is rather perplexing because it matches quite well with some of the components; for example, $\partial v / \partial y=0.08$ works quite well while others, in particular $\partial v / \partial x=3.8$, do quite poorly. The $\partial v / \partial x$ component is apparently the main trouble spot for all these models with the TRU, DUCT and $25 \mathrm{~km}$ versions

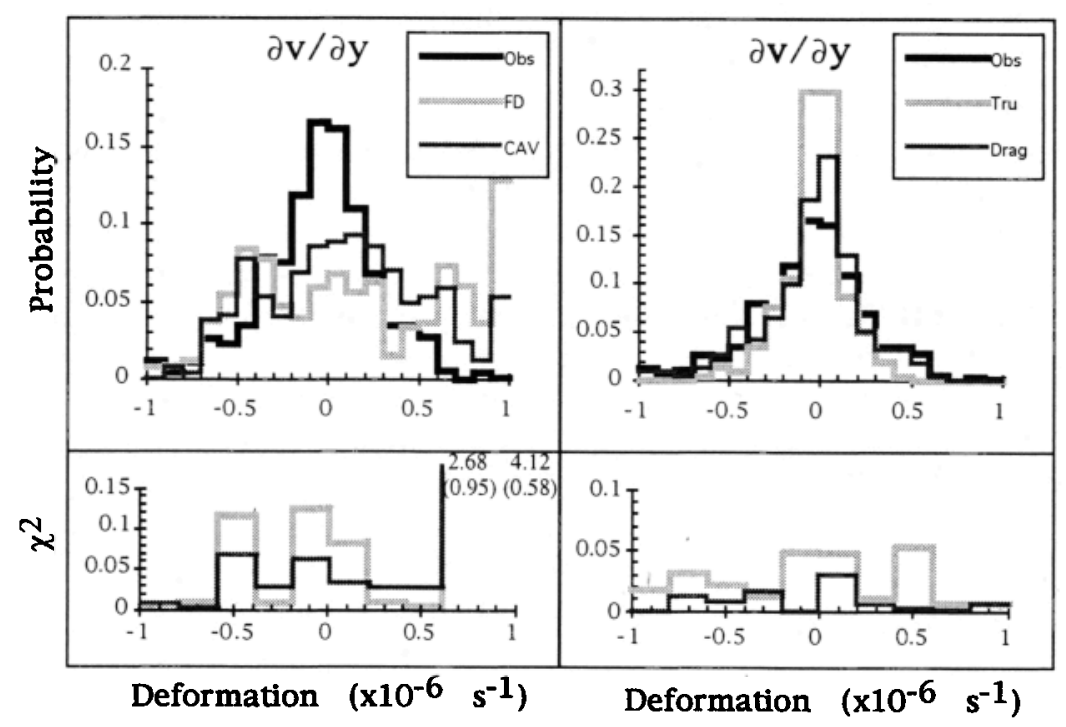

Figure 12. Example of probability distribution and corresponding $\chi^{2}$ statistics. Abbreviations are as follows: OBS, observed; FD, free drift model; CAV, cavitating fluid model; TRU, viscousplastic model with truncated ellipse; and DRAG, TRU with reduced water drag. The $\chi^{2}$ values, which are exceedingly large (i.e., off scale), are indicated numerically for FD and CAV without and with parentheses, respectively. 
Table 2. $\chi^{2}$ Results

\begin{tabular}{cccccccc}
\hline & FD & CAV & CON & TRU & DUCT & DRAG & $25 \mathrm{~km}$ \\
\hline VM & 0.28 & 0.26 & $\mathbf{0 . 4 3}$ & 0.37 & $0.15 \mathrm{~B}$ & $0.88 \mathrm{~W}$ & 0.22 \\
VD & $\mathbf{0 . 8 9} \mathrm{W}$ & 0.42 & $\mathbf{0 . 3 0}$ & $0.29 \mathrm{~B}$ & 0.35 & 0.39 & 0.35 \\
$\partial u / \partial x$ & $\mathbf{0 . 3 9} \mathrm{W}$ & 0.35 & 0.08 & 0.08 & 0.08 & 0.21 & $\mathbf{0 . 0 7} \mathrm{B}$ \\
$\partial u / \partial y$ & $\mathbf{0 . 4 1} \mathrm{W}$ & $\mathbf{0 . 1 9}$ & $\mathbf{0 . 2 1}$ & 0.21 & 0.20 & $0.15 \mathrm{~B}$ & 0.17 \\
$\partial v / \partial x$ & 1.53 & 1.30 & 1.01 & 0.76 & 0.67 & $3.84 \mathrm{~W}$ & $\mathbf{0 . 4 5} \mathrm{B}$ \\
$\partial v / \partial y$ & $7.13 \mathrm{~W}$ & 1.77 & $\mathbf{0 . 2 6}$ & 0.26 & 0.34 & $0.08 \mathrm{~B}$ & 0.25 \\
DV & $38.14 \mathrm{~W}$ & 3.07 & $\mathbf{0 . 4 1}$ & $\mathbf{0 . 4 5}$ & $\mathbf{0 . 5 9}$ & $0.15 \mathrm{~B}$ & 0.41 \\
ND & $1.37 \mathrm{~W}$ & 0.74 & $\mathbf{0 . 1 5}$ & $\mathbf{0 . 1 6}$ & $\mathbf{0 . 1 3}$ & $0.05 \mathrm{~B}$ & 0.14 \\
SD & $\mathbf{0 . 9 8} \mathrm{W}$ & 0.52 & $\mathbf{0 . 1 6}$ & $\mathbf{0 . 1 4}$ & $\mathbf{0 . 1 2} \mathrm{B}$ & $\mathbf{0 . 2 0}$ & 0.14 \\
VT & $\mathbf{0 . 5 1 \mathrm { B }}$ & $\mathbf{0 . 5 7}$ & $\mathbf{0 . 5 8}$ & 0.52 & 0.54 & $4.41 \mathrm{~W}$ & 0.53 \\
\hline
\end{tabular}

Variables were computed from observed versus simulated results from the large-scale buoy array at Ice Station Weddell in 1992 with a 30 hour low-pass filter applied. Abbreviations are as follows: FD, free drift model; $\mathrm{CAV}$, cavitating fluid model; CON, viscous-plastic model with concentric ellipse; TRU, viscous-plastic model with truncated ellipse; DUCT, TRU with reduced ductility $\zeta_{\max } \rightarrow \zeta_{\max } / 100 ;$ DRAG, TRU with reduced water drag (see text); $25 \mathrm{~km}$, TRU run at $25 \mathrm{~km}$ resolution; VM, velocity magnitude, and VD, velocity direction, both in centimeters per second; and DV, divergence; ND, normal deformation (elongation); SD, local shear; and VT, vorticity, all in units of $\times 10^{-6} \mathrm{~s}^{-1} ; \mathrm{B}$ and $\mathrm{W}$, best and worst case from each category.

improving increasingly. This component represents the shear of the $v$ velocity (parallel to the shelf break) in the $x$ direction (normal to the shelf break). This information suggests that topographically enhanced processes may be important to incorporate into the model in order to improve this situation. This is also the direction believed by Geiger et al. [1998] to be most heavily influenced by tidal amplification in the western Weddell Sea region due to a change in water depth by a factor of 2 from the western buoy sites (about $1500 \mathrm{~m}$ ) to the eastern buoy sites (about $3000 \mathrm{~m}$ ). Inclusion of subdaily processes, specifically tidal/inertial motion, is the suggested hypothesis for decreasing this largest $\chi^{2}$ result between the observed and modeled buoy deformation components.

Sorting this out by the more physically based components (differential kinematic parameters) of divergence, vorticity, normal deformation (local elongation), and local shear, we see from Table 2 that free drift and cavitating fluid have significantly greater $\chi^{2}$ values in all cases, except vorticity, which is part of the nondeformational rotational portion of the array. The reduced water drag case seems to do quite well for most of the deformation terms but not for velocity magnitude, direction, and vorticity. The remaining four (CON, TRU, DUCT, $25 \mathrm{~km}$ ) do about equally well, but each is a little different for each process.

\section{Discussion and Conclusions}

Summarizing these statistics, we find that neither free drift nor the cavitating fluid models adequately reproduces both drift and deformation features in the ice. The viscous-plastic rheology with its various modifications shows a considerable improvement over these, but none of the modifications clearly stands out as unique in terms of satisfying all the drift and deformation results. All results do show a distinct effect attributed to topography contributing to overall greater $\chi^{2}$ values and a low correlation in modeled versus observed divergence. Both of these may be improved if undamped subdaily tidal/inertial processes and possibly anisotropy are included in the models [Hibler and Schulson, 1997].

Key results seen in this comparison are as follows. First, while none of the models completely reproduces the buoy behavior observed in the field, the use of a rheology with both pressure and shear resistance is the best solution of those studied here. Second, with regard to sensitivity to these models, ice deformation is far more difficult to predict than drift and is much more sensitive in terms of response to the tuning of internal ice parameters. Third, an adequate model improving both drift and deformation was not achieved. While use of increased ductility in the viscous-plastic model greatly improved velocity magnitude statistics, modifying the drag coefficients was far more successful at improving many of the deformation components; however, even a combination of these [Geiger, 1996] failed to produce a satisfactory result. Overall, shear is reproduced much better than divergence in the models and velocity magnitude is predicted much better than direction owing to the input field quality. Fourth, the sensitivity of the different deformation terms (both directional and invariants) is such that some parameters improve while others do not, depending on the modifications made to the models. Fifth, a distinct directional dependence relative to topographic characteristics is evident in $\chi^{2}$ results, with the connection to topography being hypothesized through subdaily oceanic (tidal) processes linked to inertial oscillations in the ice. 
A salient conclusion of this work is that deformation provides a more discriminating test between different sea ice rheology models. This is apparent both in the time series of deformation from the buoy array and in the progressive vector plots of the buoys over the duration of the field experiment. The basic picture that emerges is one where low-frequency forcing is supplied by the wind, with the average ice drift following the wind forcing in a manner only weakly dependent upon internal ice stress. However, the ice interaction provides a coherence to the differential motion, so that different portions of the ice pack do not disperse and mix, which occurs if they were allowed to freely move with the wind. This ice interaction also causes a reduction in variance of the deformation in comparison to what would be expected with only wind forcing, which is consistent with observations. This can be seen both in the long-term buoy drift over the period of the experiment and in the variance of the deformation.

Comparisons of different models have shown that the main feature of a successful model is the inclusion of some level of shear strength that reduces the net shear in the system and provides a greater coherence to the differential motion. Basically the deformation variability produced by predicted drift, excluding shear strength, tends to be much larger than observed. This feature is also apparent in the probability distribution of deformation which tends to have a much more Gaussian character with the inclusion of ice interaction, therefore comparing well with observations.

While there is coherence between modeled and observed drift at lower frequencies, there is a great deal of higher-frequency motion and deformation in the observations that is not reproduced in these largely wind driven, ice dynamics models. This is, in large part, due to tidal forcing for this region that is not included in the model forcing here. However, this defect also points to the inadequacies of the present boundary layer formulation which assumes averaging over long periods compared to the inertial period, which is almost indistinguishable from the semidiurnal tidal period at this latitude. Improvements in the formulation of this coupling are currently underway.

A combination of improved input fields and boundary layer formulation may provide the means to correct some of the differences found in this comparison study. Additionally, consideration of an anistropic rheology [e.g., Hibler and Schulson, 1997] may be a viable inclusion to consider, given the improved speed of computers and the need to include more realistic subdaily processes in the models. In the meantime, the methods presented here serve as useful processing tools for analyzing and testing model modifications in a way that directly compares observed drift and deformation behavior with numerically constructed, constitutive assumptions.

While these results are only from one regional case study, they reveal that deformation is clearly a defining parameter in terms of testing ice rheologies in models. The spatial variability of the drift (i.e., the deformation) is also the variable used in the constitutive relation from which the rheology is defined. By including deformation in the validation procedure, the variability in the field drift is also taken into account. Deformation also has a more direct impact on the compactness than the drift, as it is a dominant process in determining open water fraction. In terms of heat balance, deformation is a critical parameter in terms of evolution of the field compactness and hence the growth rates and mass balance of the system. Since the shear is intrinsically connected to the physical dynamics of the divergence process, a realistic divergent-shear rheology is needed to correctly define the open water fraction in the ice. As high-resolution and eddy-resolving ocean models become more prevalent, these smaller-scale processes become increasingly important to coupled ice-ocean models.

\section{Appendix A: Correlation and Coherence of Maximum Shear}

The following describes the mathematical details used to compute the cross correlation for maximum shear and cross-spectral coherence.

\section{A.1. Correlation Coefficient}

Since maximum shear is a positive definite quantity, the usual formulation for correlation only works for the magnitude of maximum shear but not the correlation in absolute terms. The following method allows us to compute the correlation of maximum shear in absolute terms. (Repeated indices of $i$ and $j$ sum.) The quantity

$$
\left(\dot{\epsilon}_{\imath j}\right)^{2} \equiv \dot{\epsilon}_{\imath j} \dot{\epsilon}_{i j}=\dot{\epsilon}_{x x}^{2}+\dot{\epsilon}_{y y}^{2}+2 \dot{\epsilon}_{x y}^{2}
$$

is the power of the total strain rate and is invariant, as are the divergence (DV) and maximum shear (MS)

$$
\begin{aligned}
\mathrm{DV}=\dot{\epsilon}_{\mathrm{I}} & =\dot{\epsilon}_{x x}+\dot{\epsilon}_{y y}=\dot{\epsilon}_{1}+\dot{\epsilon}_{2} \\
\mathrm{MS}= \pm \dot{\epsilon}_{\mathrm{II}} & = \\
& \pm \sqrt{\left(\frac{\dot{\epsilon}_{x x}-\dot{\epsilon}_{y y}}{2}\right)^{2}+\left(\dot{\epsilon}_{x y}\right)^{2}} \\
& =\frac{\dot{\epsilon}_{1}-\dot{\epsilon}_{2}}{2}
\end{aligned}
$$

Using (A2) and (A3) we find that the total strain-rate power equals

$$
\left(\dot{\epsilon}_{i j}\right)^{2}=\frac{\mathrm{DV}^{2}}{2}+2(\mathrm{MS})^{2}
$$

Solving for maximum shear using this and the strainrate tensor in coordinate specific terms, we get

$$
(\mathrm{MS})^{2}=\frac{\left(\dot{\epsilon}_{i j}\right)^{2}}{2}-\frac{\dot{\epsilon}_{i \imath} \dot{\epsilon}_{\jmath \jmath}}{4} \text {. }
$$


Both quantities on the right-hand side are invariant (total strain rate and divergence). Furthermore, terms like $\dot{\epsilon}_{i j}^{o} \dot{\epsilon}_{i j}^{m}$ are also invariant, provided that the tensors represented by matrix superscript $o$ and superscript $m$ are both in the same coordinate system. Designating $o$ for observed and $m$ for model, we can form the following invariant correlation of maximum shear

$$
\hat{R}_{o m}[\tau]=\sum_{t=1}^{N} \frac{\dot{\epsilon}_{2 j}^{o}[t] \bar{\epsilon}_{2 j}^{m}[t+\tau]}{2}-\frac{\dot{\epsilon}_{\imath 2}^{o}[t] \epsilon_{j j}^{m}[t+\tau]}{4}
$$

where repeated subscript indices are summed over, $\tau$ is the lag time, and $N$ is the total number of data minus the number of data that lag at time $\tau$. In principal coordinate space, this reduces to

$$
\hat{R}_{o m}=\frac{\left(\dot{\epsilon}_{1}^{o}-\dot{\epsilon}_{2}^{o}\right)\left(\dot{\epsilon}_{1}^{m}-\dot{\epsilon}_{2}^{m}\right)}{4} .
$$

Consequently, the normalized correlations are given by

$$
\begin{aligned}
& \hat{\rho}_{o m}[\tau]=\frac{\hat{R}_{o m}[\tau]}{\dot{\epsilon}_{\mathrm{II}}^{o}[t=0] \dot{\epsilon}_{\mathrm{II}}^{m}[t=0]} \\
& \hat{\rho}_{o m}[\tau]=\frac{\hat{R}_{o m}[\tau]}{\sqrt{\hat{R}_{o o}[t=0]} \sqrt{\hat{R}_{m m}[t=0]}} .
\end{aligned}
$$

\section{A.2. Cross-Spectral Coherence}

The cross-spectral density function is the Fourier transform of the cross-correlation function and is defined as the complex function

$$
\begin{aligned}
G_{\imath \jmath}[f] & =C_{i \jmath}[f]-\mathbf{j} Q_{\imath j}[f] \\
G_{i \jmath}[f] & =\left|G_{\imath j}\right| e^{-\mathbf{j} \theta_{\imath \jmath}} \\
\left|G_{i j}\right| & =\sqrt{C_{\imath \jmath}^{2}+Q_{\imath \jmath}^{2}} \\
\theta_{i j} & =\tan ^{-1}\left(\frac{Q_{i \jmath}}{C_{\imath \jmath}}\right)
\end{aligned}
$$

where $C_{\imath \jmath}$ is the real or coincident spectral function, $Q_{i j}$ is the imaginary or quadrature spectral density function, and $\mathbf{j}$ is the imaginary value $\sqrt{-1}$ in Cartesian space. In polar coordinates, $\left|G_{\imath \jmath}\right|$ is the magnitude of the function and $\theta_{\imath j}$ is the phase. When normalized, the magnitude,

$$
\gamma_{\imath \jmath}^{2}[f]=\frac{\left|G_{\imath \jmath}\right|^{2}}{G_{\imath i} G_{j j}}
$$

is known as the coherence.

One way to obtain the coherence is to compute the ræal and imaginary components, following Bendat and Fiersol [1971], such that $C_{\imath j} \approx \hat{C}_{k}$ and $Q_{i j} \approx \hat{Q}_{k}$ and applying Hanning smoothing such that

$$
\begin{aligned}
\hat{C}_{0}= & 0.5 \tilde{C}_{0}+0.5 \tilde{C}_{1} \\
\hat{C}_{k}= & 0.25 \tilde{C}_{k-1}+0.5 \tilde{C}_{k}+0.25 \tilde{C}_{k+1} \\
& k=1, \ldots, m-1 \\
\hat{C}_{m}= & 0.5 \tilde{C}_{m-1}+0.5 \tilde{C}_{m} .
\end{aligned}
$$

for both $\hat{C}_{k}$ and $\hat{Q}_{k}$ where

$$
\begin{aligned}
\tilde{C}_{k}[f]= & 2 \Delta t \\
& \left(\hat{A}_{0}+2 \sum_{r=1}^{m-1} \hat{A}_{r} \cos \left[\frac{\pi r k}{m}\right]+(-1)^{k} \hat{A}_{m}\right) \\
\bar{Q}_{k}[f]= & 4 \Delta t \sum_{r=1}^{m-1} \hat{B}_{r} \sin \left[\frac{\pi r k}{m}\right]
\end{aligned}
$$

such that $f=k f_{c} / m$ is the given frequency for $k=$ $0,1, \ldots, m$ and $f_{c}=1 / 2 \Delta t$ is the cutoff/Nyquist frequency. In this way, we separate the cross-correlation into even and odd parts defined by

$$
\begin{aligned}
& \hat{A}_{r}=\hat{A}_{\imath \jmath}[\tau]=\frac{1}{2}\left(\hat{R}_{i j}+\hat{R}_{j i}\right) \\
& \hat{B}_{r}=\hat{B}_{i \jmath}[\tau]=\frac{1}{2}\left(\hat{R}_{\imath \jmath}-\hat{R}_{j \imath}\right) .
\end{aligned}
$$

\section{Appendix B: Numerical Method for Plastic Solution}

The momentum balance for sea ice is used to solve ice velocity, given known air and ocean inputs. For a nonlinear viscous-plastic rheology [Hibler, 1979] and energy-conserving variations as constructed by $I p$ [1993] and Hibler and Ip [1995], the ice rheological parameters of pressure $P$, bulk $\zeta$, and shear $\eta$ viscosities are all interlinked functions of the strain-rate invariants. Consequently, for a true plastic solution of the nonlinear equations of motion, we need to solve for these linked nonlinear parameters by some iterative process.

The efficient numerical method by Zhang and Hibler [1997] (hereinafter referred to as ZH) solves the linearized equations of motion (i.e., $D \mathbf{v} / d t \rightarrow \partial \mathbf{v} / \partial t$ ) in a decoupled form through a matrix solution (Thomas algorithm), together with line successive over relaxation (SOR). Building on their method, we describe here a method to efficiently solve the ice velocities by a pseudo time stepping procedure where we subcycle the equations of motion at each time step to approach plastic flow.

For simplicity, we reorganize (2a) and (2b) of $\mathrm{ZH}$ to

$$
\begin{aligned}
& \left(\delta_{t}+\delta_{u x}+A_{w}\right) u=\left(\delta_{v x}+B_{w}\right) v+\tau_{x} \\
& \left(\delta_{t}+\delta_{v y}+A_{w}\right) v=\left(\delta_{u y}-B_{w}\right) u+\tau_{y}
\end{aligned}
$$

where the $\delta$ terms are the operators

$$
\begin{aligned}
\delta_{t} & =\rho_{i} \vec{h} \frac{\partial}{\partial t} \\
\delta_{u x} & =-\frac{\partial}{\partial x}(\zeta+\eta) \frac{\partial}{\partial x}-\frac{\partial}{\partial y} \eta \frac{\partial}{\partial y} \\
\delta_{v y} & =-\frac{\partial}{\partial y}(\zeta+\eta) \frac{\partial}{\partial y}-\frac{\partial}{\partial x} \eta \frac{\partial}{\partial x} \\
\delta_{v x} & =\frac{\partial}{\partial x}(\zeta-\eta) \frac{\partial}{\partial y}+\frac{\partial}{\partial y} \eta \frac{\partial}{\partial x} \\
\delta_{u y} & =\frac{\partial}{\partial y}(\zeta-\eta) \frac{\partial}{\partial x}+\frac{\partial}{\partial x} \eta \frac{\partial}{\partial y} .
\end{aligned}
$$


All the external and internal nonice velocity terms are described by

$$
\begin{aligned}
& \tau_{x}=A_{a} U_{a}-B_{a} V_{a}-\frac{\partial P}{\partial x} \\
& \tau_{y}=A_{a} V_{a}+B_{a} U_{a}-\frac{\partial P}{\partial y}
\end{aligned}
$$

with the nonlinear air and water drag coefficients described by

$$
\begin{aligned}
A_{a} & =C_{a}^{*} \rho_{a}\left|\mathbf{V}_{\mathbf{a}}\right| \cos \theta_{a} \\
B_{a} & =C_{a}^{*} \rho_{a}\left|\mathbf{V}_{\mathbf{a}}\right| \sin \theta_{a} \\
A_{w} & =C_{w}^{*} \rho_{w}\left|\mathbf{v}-\mathbf{V}_{\mathbf{w}}\right| \cos \theta_{w} \\
B_{w} & =C_{w}^{*} \rho_{w}\left|\mathbf{v}-\mathbf{V}_{\mathbf{w}}\right| \sin \theta_{w}+\rho_{i} f
\end{aligned}
$$

The $C^{*}$ terms are the constants referred to in the text as drag coefficients; subscripted variables $i, a$, and $w$ are for ice, air, and water, respectively; $f$ is the Coriolis parameter; and $P, \zeta$, and $\eta$ are the internal ice parameters computed as described in Appendix C. All these terms are further described by Hibler [1979]. The coefficients $A_{w}$ and $B_{w}$ are the diagonal and cross-diagonal terms $C_{d}$ and $C_{s}$, respectively, defined by ZH. Of paramount importance to the method described here is the inclusion of $\mathbf{v}$ in the water drag terms $A_{w}$ and $B_{w}$.

Using a finite difference scheme with Arakawa B grid, nonlinear viscosity $(\zeta, \eta)$, internal ice pressure $P$, and other scalar properties are defined in the center of each grid cell. All vector components, including the ice velocities $(u, v)$ and all derived vector quantities, such as the internal ice pressure gradient and scalar quantities multiplied by velocity, such as the mean ice thickness $\bar{h}$ and Coriolis parameter $f$, are located in the corners of the grid cells. Tensor quantities are centered at scalar locations, with gradient operations computed using a five-point differencing scheme for $\delta_{u x}$ and $\delta_{v y}$ and a nine-point differencing scheme for $\delta_{v x}$ and $\delta_{u y}$ after Hibler [1979].

Our main concern here is the time-stepping procedure consisting of a physical time step with subcycles of pseudo time steps nested around a predictor-corrector cycle. Within each predictor and each corrector is a matrix solver, which itself contains the SOR relaxation iterations. Hence there are four nested steps needed to achieve the full viscous-plastic solution, with the outermost loop being the physical time step. Here $\delta_{t}$ is the only term containing velocity from the previous physical time step $k$ because it contains no other velocity dependent terms.

In the absence of a predictor-corrector scheme, time stepping for (B1) (and analogously for (B2)) would be

$$
\begin{array}{r}
{\left[\frac{\rho \bar{h}}{\Delta t}+\delta_{u x}\left(\mathbf{v}^{(n)}\right)+A_{w}\left(\mathbf{v}^{(n)}\right)\right] u^{(n+1)}} \\
=\left[\delta_{v x}\left(\mathbf{v}^{(n)}\right)+B_{w}\left(\mathbf{v}^{(n)}\right)\right] v^{(n)} \\
+\tau_{x}\left(\mathbf{v}^{(n)}\right)-\frac{\rho \bar{h}}{\Delta t} u^{(k)}
\end{array}
$$

Here superscript $k$ represents physical time steps while $n$ represents the pseudo time step such that $u^{(k+1)}=$ $u^{(n+1)}$ at the end of each physical time step and $u^{(k)}=$ $u^{(n=1)}$ at the beginning of the first pseudo time step. The full plastic solution is reached when $u^{(n+1)}-u^{(n)}<$ $\epsilon$. For each pseudo time step, the SOR matrix solver in ZH steps through $i$ iterations until $u^{(i+1)}-u^{(i)}<\epsilon$ such that $u^{(n+1)}=u^{(i+1)}$. In this form, the $(n+1)$ superscript represents the velocity updated to the most recent matrix solution. All the variables dependent on the ice velocity, such as $\zeta, \eta, P, A_{w}$, and $B_{w}$, are computed from the previous pseudo time step solution $u^{(n)}$.

The solution above would be stable, were it not for the nonlinear dependence of the water drag on the ice velocity (i.e., the $\mathbf{v}$ term noted in $A_{w}$ and $B_{w}$ ). Because of this ice velocity dependence, the solution requires a predictor-corrector scheme (also called a modified Euler scheme) embedded within each pseudo time step in order to stably adjust to these nonlinearities. To do this, the input velocity in the predictor scheme is the most recently updated one while in the corrector mode, the velocity in $A_{w}, B_{w}$ and the velocity multiplied by $\delta_{v x}^{(n)}$, are averages from the previous pseudo time step and most recent predictor results.

In this way, (B14) is modified into a two-part predictorcorrector procedure, with the predictor part computed by

$$
\begin{gathered}
{\left[\frac{\rho \bar{h}}{\Delta t}+\delta_{u x}\left(\mathbf{v}^{(n)}\right)+A_{w}\left(\mathbf{v}^{(n)}\right)\right] u^{(n+1) *}} \\
=\left[\delta_{v x}\left(\mathbf{v}^{(n)}\right)+B_{w}\left(\mathbf{v}^{(n)}\right)\right] v^{(n)} \\
+\tau_{x}\left(\mathbf{v}^{(n)}\right)-\frac{\rho \bar{h}}{\Delta t} u^{(k)}
\end{gathered}
$$

with a similar formulation computed to obtain $v^{(n+1) *}$. Note that this predictor part has the same form as (B14).

Following this, we include the corrector step

$$
\begin{array}{r}
{\left[\frac{\rho \bar{h}}{\Delta t}+\delta_{u x}\left(\mathbf{v}^{(n+1) *}\right)+A_{w}\left(\mathbf{v}^{(c)}\right)\right] u^{(n+1) * *}} \\
=\left[\delta_{v x}\left(\mathbf{v}^{(n+1) *}\right)+B_{w}\left(\mathbf{v}^{(c)}\right)\right] v^{(c)} \\
+\tau_{x}\left(\mathbf{v}^{(c)}\right)-\frac{\rho \bar{h}}{\Delta t} u^{(k)}
\end{array}
$$

and analogously solve for $v^{(n+1) * *}$, where

$$
\mathbf{v}^{(c)}=\frac{\mathbf{v}^{(n)}+\mathbf{v}^{(n+1) *}}{2}
$$

Note that $\zeta, \eta$, and $P$ are updated before each matrix solution, while the nonlinear water drag advances more slowly to prevent instabilities.

To remain consistent with $\mathrm{ZH}$, a Coriolis correction is carried out to solve for $\mathbf{v}^{(n+1)}$ utilizing both component equations such that

$$
\bar{A}_{w}\left(\mathbf{v}^{(c)}\right) u^{(n+1)}-B_{w}\left(\mathbf{v}^{(c)}\right) v^{(n+1)}=\tilde{\tau}_{x}
$$




$$
\tilde{A}_{w}\left(\mathbf{v}^{(c)}\right) v^{(n+1)}+B_{w}\left(\mathbf{v}^{(c)}\right) u^{(n+1)}=\tilde{\tau}_{y}
$$

where $\tilde{\tau}_{x}$ and $\tilde{\tau}_{y}$ are the terms from the corrector equation expressed as

$$
\begin{gathered}
\tilde{\tau}_{x}=\delta_{v x}\left(\mathbf{v}^{(n+1) *}\right) v^{(c)}-\delta_{u x}\left(\mathbf{v}^{(n+1) *}\right) u^{(n+1) * *} \\
+\tau_{x}\left(\mathbf{v}^{(c)}\right)-\frac{\rho \bar{h}}{\Delta t} u^{(k)} \\
\tilde{\tau}_{y}=\delta_{u y}\left(\mathbf{v}^{(n+1) *}\right) u^{(c)}-\delta_{v y}\left(\mathbf{v}^{(n+1) *}\right) v^{(n+1) * *} \\
+\tau_{y}\left(\mathbf{v}^{(c)}\right)-\frac{\rho \bar{h}}{\Delta t} v^{(k)}
\end{gathered}
$$

and for brevity,

$$
\tilde{A}_{w}=\frac{\rho \bar{h}}{\Delta t}+A_{w}\left(\mathbf{v}^{(c)}\right)
$$

In this form, the Coriolis adjustment can be computed by simultaneously solving (B18) and (B19) to form the simple algebraic result

$$
\begin{aligned}
u^{(n+1)} & =\frac{\tilde{A}_{w} \tilde{\tau}_{x}+B_{w} \tilde{\tau}_{y}}{\tilde{A}_{w}^{2}+B_{w}^{2}} \\
v^{(n+1)} & =\frac{\tilde{A}_{w} \tilde{\tau}_{y}-B_{w} \tilde{\tau}_{x}}{\tilde{A}_{w}^{2}+B_{w}^{2}} .
\end{aligned}
$$

Experience has shown that the most consistent approach is to apply this Coriolis adjustment at the ends of both the predictor and corrector steps.

This procedure can be contrasted to the normal modified Euler pseudo time stepping procedure where the viscous operators $\delta_{u x}\left(\mathbf{v}^{(n+1) *}\right)$ and $\delta_{v x}\left(\mathbf{v}^{(n+1) *}\right)$ in (B16) (B20), and (B21) are replaced by $\delta_{u x}\left(\mathbf{v}^{(c)}\right)$ and $\delta_{v x}\left(\mathbf{v}^{(c)}\right)$.

The following summarizes our approach to the full plastic solution. One predictor-corrector loop within each time step solves for the ice velocity for given internal ice parameters of $P, \zeta$, and $\eta$. Since the internal ice pressure and viscosities are functions of the ice velocity, the pressure and viscosity terms in both the predictor and corrector steps use the most currently known ice velocity. The values of these terms are changing each time they are computed, in response to both the new velocities computed and the force balance. Creating a loop of pseudo time steps inside each physical time step but around each predictor-corrector pair allows the velocities, internal ice pressure, and viscosities to be updated each time the force balance routine and relaxation combination is called. Additionally, the nonlinear water drag is updated for each pseudo time step.

The end result is an iterative convergence toward a force balance based on one velocity field. The impact of pseudo time stepping on the precision of deformation components is most clearly seen in Figure 10 of $\mathrm{ZH}$. Unlike $\mathrm{ZH}$, we update the viscosities and ice pressure at each of the predictor and corrector steps. In this way, we reach a plastic solution close to that of 100 pseudotimes in the ZH method, but in about 10 pseudo time steps (see Figure 9).

\section{Appendix C: Constitutive Law Equations}

The rheologies examined here are basically variations on an elliptical yield curve with modifications, for example, to ensure energy conservation $[I p, 1993 ;$ Hibler and Ip, 1995] and to remove tensile stress [Hibler and Schulson, 1997]. For an elliptical yield curve following Hibler [1979], the stress is given by

$$
\sigma_{i j}=2 \eta \dot{\epsilon}_{i j}+(\zeta-\eta) \dot{\epsilon}_{k k}-P / 2
$$

where repeated subscripts are summed over and $\zeta$ and $\eta$ are the bulk and shear viscosities, respectively, expressed as functions of the strain rate according to

$$
\begin{aligned}
& \zeta=P_{\max } /(2 \Delta) \\
& \eta=\zeta / e^{2} \\
& \Delta=
\end{aligned}
$$

$\sqrt{\left(\dot{\epsilon}_{11}^{2}+\dot{\epsilon}_{22}^{2}\right)\left(1+1 / e^{2}\right)+4 \dot{\epsilon}_{12}^{2} / e^{2}+2 \dot{\epsilon}_{11} \dot{\epsilon}_{22}\left(1-1 / e^{2}\right)}$

Here $P_{\max }$ is the maximum ice strength (equal to the ice pressure $P$ for high strain rates) and $e$ is a constant equal to 2 for the elliptical yield curve chosen for sea ice. The maximum ice pressure $P_{\max }$ is related to the mean ice thickness per unit area $h$ and the ice compactness $A$ by $P_{\max }=P^{*} h \exp \{-C(1-A)\}$, where $P^{*}$ and $C$ are empirical constants with values of $27.5 \times 10^{3} \mathrm{~N}$ $\mathrm{m}^{-2}$ and 20, respectively. The mean ice thickness $h$ and compactness $A$ evolve according to conservation equations described by Hibler [1979]. To approximate rigid stress states inside the yield curve, $\zeta$ and $\eta$ are capped at some large maximum value $\left(\zeta_{\max }=P_{\max } / \Delta^{*}\right.$ and $\eta_{\max }=\zeta_{\max } / e^{2}$ for $\left.\Delta^{*}=2 \times 10^{4} / 5 \times 10^{12}\right)$ for small strain rates.

To obtain a yield curve as pictured in Figure 4e, we take $\eta$ to be given by the minimum value of two quantities:

$$
\eta=\min \left[\zeta / e^{2}, \eta_{1}\right]
$$

where

$$
\begin{aligned}
\eta_{1} & =\left(P / 2-\zeta \dot{\epsilon}_{k k}\right) / \dot{\epsilon}_{s} \\
\dot{\epsilon}_{k k} & =\dot{\epsilon}_{11}+\dot{\epsilon}_{22} \\
\dot{\epsilon}_{s} & =\sqrt{\left(\dot{\epsilon}_{11}-\dot{\epsilon}_{22}\right)^{2}+4 \dot{\epsilon}_{12}^{2}}
\end{aligned}
$$

where $\dot{\epsilon}_{k k}$ and $\dot{\epsilon}_{s}$ are the divergence rate and maximum shear rate, respectively. Restricting $\eta$ to be less than $\eta_{1}$ insures that there is no tensile stress. In addition, to insure that there is no stress at zero strain rates, we insist that $P=2 \Delta \zeta_{1}$, where $\zeta_{1}=\min \left[P_{\max } / 2 \Delta, \zeta_{\max }\right]$. When plastic flow is occurring, $P$ will be a constant $\left(P_{\max }\right)$, but for $\zeta=\zeta_{\max }$, the stress state will lie on a smaller but geometrically similar yield curve (see dashed lines in Figure 4e) going through the origin.

Acknowledgments. This work was supported by funding from NSF OPP-92034700, ONR assert grant N00014-961-0615, and USRA contract NAS-5-32484. Thanks go to the 
anonymous reviewers whose comments led to improvements in the paper.

\section{References}

Bendat, J. S., and A. G. Piersol, Random Data: Analysis and Measurement Procedures, 407 pp., John Wiley, New York, 1971.

Fischer, H., and P. Lemke, On the required accuracy of atmospheric forcing fields for driving dynamic-thermodynamic sea ice models, in The Polar Oceans and Their Role in Shaping the Global Environment: The Nansen Centennial Volume, Geophys. Monogr. Ser., vol. 85, edited by $\mathrm{O}$. M. Johannessen, R. D. Muench, and J. E. Overland, pp. 373-381, AGU, Washington, D. C., 1994.

Flato, G., and W. D. Hibler III, Modeling pack ice as a cavitating fluid, J. Phys. Oceanogr., 22(6), 626-651, 1992.

Geiger, C. A., Investigation of dynamic sea ice processes in the Weddell Sea during 1992, Ph.D. thesis, 387 pp., Dartmouth Coll., Hanover, N.H., May 1996.

Geiger, C. A., S. F. Ackley, and W. D. Hibler III, Year-round pack ice in the Weddell Sea: Response and sensitivity to atmospheric and oceanic forcing, Ann. Glaciol., 25, 269$275,1997$.

Geiger, C. A., S. F. Ackley, and W. D. Hibler III, Sea ice drift and deformation processes in the western Weddell Sea, in Antarctic Sea Ice: Physical Processes, Interactions and Variability, Antarct. Res. Ser., vol. 74, edited by M.O. Jeffries, pp. 141-160, AGU, Washington, D.C., 1998.

Gordon, A. L., and B. A. Huber, Southern ocean winter mixed layer, J. Geophys. Res., 95, 11,655-11,672, 1990.

Gordon, A. L., and Ice Station Weddell Group of Principal Investigators and Chief Scientists, Weddell Sea Exploration from Ice Station, EOS, Trans., $A G U, 74$ (11), 121, 124-126, 1993.

Hibler, W. D., III, A dynamic-thermodynamic sea ice model, J. Phys. Oceanogr., 9(4), 815-846, 1979.

Hibler, W. D., III, and S. F. Ackley, Numerical simulation of the Weddell sea pack ice, J. Geophys. Res., 88, 28732887, 1983.

Hibler, W. D., III, and C. F. Ip, The effect of sea ice rheology on Arctic buoy drift, in Ice Mechanics, Am. Soc. of Mech. Eng., New York, vol. 204, edited by J. P. Dempsey and Y. D. S. Rajapakse, pp. 255-264, AMD, Vol. 204, 1995.

Hibler, W. D., III, and E. M. Schulson, On modeling sea ice fracture and flow in numerical investigations of climate, Ann. Glaciol. 25, 26-32, 1997.
Hibler, W. D., III, W. F. Weeks, A. Kovacs, and S. F. Ackley, Differential sea-ice drift, 1, Spatial and temporal variations in sea-ice deformation, J. Glaciol., 13(69), 437-455, 1974.

Hibler, W. D., III, P. Heil, and V. I. Lytle, On simulating high frequency variability in Antarctic sea-ice dynamics models, Ann. Glacıol., 27, in press, 1998.

Hines, W. W., and D. C. Montgomery, Multiple regression, in Probabiluty and Statistics in Engineering and Management Science 3rd edition, chap. 15, pp. 487-558, John Wiley, New York, 1990.

Ip, C. F., Numerical investigation of different rheologies on sea ice dynamics, Ph.D. thesis, 242 pp., Dartmouth Coll., Hanover, N.H., May 1993.

Lytle, V., and S. F. Ackley, Heat flux through sea ice in the western Weddell Sea, J. Geophys. Res., 101, 8853-8868, 1996.

Maykut, G. A., The surface heat and mass balance, in The Geophysics of Sea Ice, NATO ASI Ser., edited by N. Untersteiner, pp. 395-464, Plenum, New York, 1986.

Olbers, D., V. Gouretski, G. Seiß, and J. Schroter, $H y$ drographic Atlas of the Southern Ocean, Alfred-WegenerInst., Bremerhaven, Germany, 1992.

Parkinson, C. L., and W. M. Washington, A large-scale numerical model of sea ice, J. Geophys. Res., 84, 311-337, 1979.

Taljaard, J. J., H. van Loon, H. L. Crutcher, and R. L. Jenne, Climate of the upper air, I, Southern hemisphere, in Temperature, Dew Points, and Heights at Selected Pressure Levels, vol. 1, NAVAIR Rep. 50-IC-55, pp. 1-135, U.S. Nav. Weather Serv., Command, Washington, D.C., 1969.

Zhang, Y., and W.D. Hibler III, On an efficient numerical method for modeling sea ice dynamics, J. Geophys. Res., 102, 8691-8702, 1997.

S. F. Ackley, Snow and Ice Branch, Cold Regions Research and Engineering Laboratory, 72 Lyme Road, Hanover, NH 03755. (e-mail: sackley@crrel.usace.army.mil)

C. A. Geiger, University Space Research Association, Oceans and Ice Branch, NASA, Goddard Space Flight Center, Code 971, Greenbelt, MD 20771. (e-mail: Cathleen.A.Geiger.1@gsfc.nasa.gov)

W. D. Hibler III, Thayer School of Engineering, Dartmouth College, 8000 Cummings, Hanover, NH 03755.

(Received May 7, 1997; revised February 16, 1998; accepted February 23, 1998.) 